Implementation Intention and Action Planning Interventions in Health Contexts: State of the Research and Proposals for the Way Forward

This is the peer reviewed version of the cited article. This article may be used for noncommercial purposes in accordance with Wiley Terms and Conditions for Self-Archiving at http://olabout.wiley.com/WileyCDA/Section/id-820227.html\#terms Citation: Hagger, M. S., \& Luszczynska, A. (2014). Implementation intention and action planning Interventions in health contexts: State of the research and proposals for the way forward. Applied Psychology: Health and Well-Being, 6(1), 1-47. doi: 10.1111/aphw.12017 


\begin{abstract}
The purpose of this paper is to provide an overview of the literature on two planning intervention techniques in health behaviour research, implementation intentions and action planning, and to develop evidence-based recommendations for effective future interventions and highlight priority areas for future research. We focused our review on four key areas: (1) definition and conceptualisation; (2) format and measurement; (3) mechanisms and processes; and (4) design issues. Overall, evidence supports the effectiveness of planning interventions in health behaviour with advantages including low cost and response burden. There is, however, considerable heterogeneity in the effects across studies and relatively few registered randomised trials that include objective behavioural measures. Optimally-effective planning interventions should adopt 'if-then' plans, account for salient and relevant cues, include examples of cues, be guided rather than user-defined, and include boosters. Future studies should adopt randomised controlled designs, report study protocols, include fidelity checks and relevant comparison groups, and adopt long-term behavioural follow-up measures. Priority areas for future research include the identification of the moderators and mediators of planning intervention effects. Future research also needs to adopt 'best practice' components of planning interventions more consistently to elucidate the mechanisms and processes involved.
\end{abstract}

Key words: implementation intention, action planning, coping planning, behaviour change techniques, health behaviour. 


\section{Implementation Intention and Action Planning Interventions in Health Contexts: State of the Research and Proposals for the Way Forward}

There has been a rapid increase in research on the effects of planning interventions to promote health-related behaviour (Abraham, Kok, Schaalma, \& Luszczynska, 2011). Planning interventions are seen as important intervention techniques in their own right as well as components of more elaborate health promotion programs incorporating multiple techniques for complex health behaviour change (Bartholomew, Parcel, Kok, Gottlieb, \& Fernández, 2011; Green \& Kreuter, 2004). The proliferation of planning interventions has been largely driven by formative research adopting social-cognitive theories and models (von Suchodoletz \& Achtziger, 2011), many of which conceptualise intentions as the primary determinant of the uptake and maintenance health behaviour (Ajzen, 1985; Armitage \& Conner, 2000; Rogers, 1975; Rosenstock, 1974), and as a direct response to the considerable literature which has recognised the limitations of intentions as a predictor of behaviour (Dekker, 2008; Sheeran, 2002; Webb \& Sheeran, 2006). The so-called intention-behaviour 'gap', that is, the imperfect relation between intentions to perform a particular behaviour and actual behavioural engagement, is frequently cited as a conceptual limitation of social-cognitive theories, despite numerous attempts to provide explanations for the inconsistency through key moderator variables (Godin, Conner, \& Sheeran, 2005; Hagger, 2010a; Scholz, Schuz, Ziegelmann, Lippke, \& Schwarzer, 2008). Volitional planning interventions are a promising avenue of inquiry to resolve this contentious issue in theory-based research on health behaviour change (Gollwitzer \& Sheeran, 2006; Schwarzer, 2001; Sheeran, Milne, Webb, \& Gollwitzer, 2005). Prominent among these planning interventions are implementation intention and action planning $^{1}$ techniques. The planning techniques, involving a variety of formats ranging from free-response to volitional planning sheets, aim to bolster or augment intentions with means to promote recall and enactment of the intended behaviour.

Implementation intentions and action planning are two of the most recognised and frequently-applied planning techniques adopted to change health behaviour (Adriaanse,

\footnotetext{
${ }^{1}$ In the current article, we use the term planning interventions or planning techniques to mean interventions that adopt implementation intentions or action planning components or techniques.
} 
Vinkers, De Ridder, Hox, \& De Wit, 2011; Bélanger-Gravel, Godin, \& Amireault, 2013; Webb, Sniehotta, \& Michie, 2010). A cursory search of the literature reveals that the number of articles making explicit reference to, or use of, implementation intention and action planning approaches in the health domain has increased more than ten-fold in the past decade from 63 articles in 2001 to 849 articles to September $2012^{2}$. There are numerous reasons why these approaches have attracted so much attention in the literature: (1) they are steeped in established social psychological theory, have been embedded in popular and well-cited theories of social cognition applied in health contexts such as the theory of planned behaviour ${ }^{3}$, and address a commonly-known limitation of these theories (i.e., the intention-behaviour 'gap'); (2) they have intuitive appeal in their parsimony; (3) they have low response burden making their promulgation through multiple modes of delivery comparatively easy; and (4) they are lowcost. Above all, there is growing support for their effectiveness in engendering behaviour change health-related contexts as stand-alone intervention strategies or as part of more elaborate interventions involving multiple behaviour-change techniques. Implementation intention and action planning interventions have been shown to be effective in changing diverse behaviours such as physical activity (Arbour \& Martin Ginis, 2009; Barg et al., 2012; Conner, Sandberg, \& Norman, 2010; Gellert, Ziegelmann, Lippke, \& Schwarzer, 2012; Luszczynska, 2006; Milne, Orbell, \& Sheeran, 2002; Prestwich et al., 2012; Prestwich, Lawton, \& Conner, 2003), healthy and unhealthy eating (Adriaanse, de Ridder, \& de Wit, 2009; Adriaanse et al., 2010; Armitage, 2007; Chapman, Armitage, \& Norman, 2009; Prestwich, Ayres, \& Lawton, 2008; Sullivan \& Rothman, 2008), smoking (Armitage, 2008; Armitage \& Arden, 2008), alcohol consumption (Armitage, 2009; Hagger, Lonsdale, Koka, et al., 2012), breast self-examination (Orbell, Hodgkins, \& Sheeran, 1997; Prestwich et al., 2005), rehabilitation from injury (Scholz, Sniehotta, Schuz, \& Oeberst, 2007), vitamin consumption (Sheeran \& Orbell, 1999b), sun-safety behaviours (e.g., Craciun, Schuz, Lippke, \& Schwarzer,

\footnotetext{
${ }^{2}$ Based on a search of the Web of Science (Thomson ISI, 2012) database for published items from January 2001 to September 2012 and with search terms "implementation intentions" or "action planning" with "health", excluding duplicates and items using these terms to mean something other than planning interventions.

${ }^{3}$ Planning approaches have been integral to newly-developed social-cognitive models of health-related behaviour such as the Health Action Process Approach (HAPA).
} 
2012), cancer screening behaviours (Browne \& Chan, 2012; Rutter, Steadman, \& Quine, 2006; Sheeran \& Orbell, 2000), workplace health and safety (Sheeran \& Silverman, 2003), vaccine uptake (Milkman, Beshears, Choi, Laibson, \& Madrian, 2011; Payaprom, Bennett, Alabaster, \& Tantipong, 2011), contraception use (de Vet et al., 2011; Martin, Sheeran, Slade, Wright, \& Dibble, 2009; Teng \& Mak, 2011), and dental health behaviours (Orbell \& Verplanken, 2010; Schuz, Wiedemann, Mallach, \& Scholz, 2009).

Previous systematic reviews have analysed the impact of implementation intentions on behaviour in multiple behavioural domains (Gollwitzer \& Sheeran, 2006) and, in specific health-related behavioural domains such as physical activity (Bélanger-Gravel et al., 2013; Carraro \& Gaudreau, 2013) and healthy eating (Adriaanse, Vinkers, et al., 2011). While these reviews report significant overall effect sizes of medium magnitude for planning interventions on behaviour, particularly for implementation intentions, they also identify substantial heterogeneity in the effect sizes across studies, particularly for research including longer-term follow-up measures of behavioural outcomes, which remains unexplained by moderator variables. A shortcoming of these analyses is that they include research that is considered of lower quality according to CONSORT guidelines such as non-randomised trials and those that have not been formally registered a priori on a recognised trials database. The application of such stringent quality criteria would likely eliminate the majority of tests of the effects from these analyses, a fact that clearly indicates the need for better quality research in the field. In addition, the reviews also include studies of a relatively low methodological quality. In fact, few studies adopting planning techniques in the health domain have paid due consideration to quality issues in measurement and methodological standards (see Conner et al., 2010, and De Vet et al., 2009, as examples of studies that have included objective measures of behaviour and trial registration, respectively). The substantial heterogeneity in effect sizes of the previous reviews of planning interventions may also stem from shortcomings specific to the planning interventions, such as variation in the definition and operationalisation of planning procedures. This heterogeneity presents considerable challenges when attempting to systematically evaluate of the evidence in terms of the effectiveness of planning interventions in health behavioural contexts. 
The problems of heterogeneity in the effect sizes as well as a recognised lack of consensus in the definitions and operationalisation of planning interventions in health contexts present considerable problems to researchers attempting to develop interventions to change health behaviour adopting planning techniques. In the current article we aim to provide a clear position statement on the state of the research on planning interventions in the health literature based on a comprehensive and critical review of the current evidence. Given the proliferation of research adopting planning techniques, there is a need to establish consensus of the most effective means to implement and evaluate planning interventions in order to move the field forward and resolve some of the operational and methodological shortcomings of previous research. Specifically, our aims are to discuss the research on interventions adopting planning components; identify their common features and differences in terms of operationalisation, design, measurement, mechanisms, and evaluation of planning components; identify the salient gaps in the literature; formulate guidelines for good practice; and, most importantly, identify priority areas for high-quality future research that will improve understanding of planning interventions in the field of health behaviour.

We focus our discussion on four emerging areas in the theoretical and empirical literature on planning interventions in health contexts: (1) definition and conceptualisation of planning interventions (e.g., distinction between types of planning intervention and their role in social-cognitive models); (2) format and measurement of planning manipulations (e.g., mode of delivery, measurement effects, format, use of examples, self- vs. other-defined plans), (3) moderators, mechanisms and processes (e.g., the role of habit, moderators of planning intervention effects, forming multiple plans, planning interventions for low intenders), and (4) design issues (e.g., sustainability of behaviour change, intervention fidelity). We will then draw from this evidence to form guidelines for best practice and recommendations for future research endeavour. The key issues, identified in this position paper, and research priorities which may be crucial for obtaining further advancements in field are displayed in Table 1. Although there are several papers providing an overview of the role of planning in the context of health behaviour (e.g., Adriaanse, Vinkers, et al., 2011; Sniehotta, 2009), the existing reviews do not provide thorough overviews of definitions, format, mechanisms, and formal 
aspects (design, format and measurement) of planning interventions in health behaviour contexts. Our position paper aims to fill this void.

\section{Definition and Conceptualisation}

We begin with an overview of the definitions and theoretical underpinnings of implementation intentions and action planning. The aim is to provide a brief overview of the theoretical origins of the two techniques and discuss current thinking on the mechanisms and processes by which they work. Importantly, we critically evaluate the validity of making a distinction between implementation intentions and action planning. We then move on to discuss how planning components have been embedded in the social-cognitive models that have been typically applied to explain and change health-related behaviour and how such an endeavour has furthered understanding of the processes involved and helped advance the understanding of health behaviour and intervention design.

\section{Are Implementation Intentions and Action Planning Distinct Techniques?}

The use of planning techniques in the domain of health and behaviour change is not a new phenomenon. Planning, as a broad concept, incorporates numerous frequently used and recognised approaches in the behavioural health literature including goal setting (e.g., Bagozzi \& Edwards, 1998) and action planning (Leventhal, Singer, \& Jones, 1965). The two categories of planning on which we focus in the current review, implementation intentions and action planning, share many characteristics of these previous approaches and have common roots in Lewin's (1951) action research model. Recent conceptualisations of planning techniques have advanced thinking and catalysed research on their use in health contexts by clearly specifying their content and mechanisms in a formal model proposed by Gollwitzer and colleagues (Gollwitzer, 1999; Gollwitzer \& Sheeran, 2006; Heckhausen \& Gollwitzer, 1987). The basic components of planning from this perspective are encapsulated in Gollwitzer's implementation intention concept. Action planning tends to incorporate the same basic building blocks as implementation intentions to the extent that, in many cases, the two approaches are indistinguishable in content. This has led researchers to use the terms implementation intentions and action planning synonymously. However, in other cases researchers have made a distinction between the two approaches in terms of the focus, number of components, and 
theoretical and operational underpinning. In this section we analyse the defining characteristics of these two approaches to planning and identify their shared and divergent attributes.

Implementation intentions were originally defined as 'if-then' plans aimed at forging a link between a critical, unconditional situation with a goal-directed behavioural response (Gollwitzer, 1999; Gollwitzer \& Sheeran, 2006). In contrast to intentions, which focus on desired outcomes or end-states (i.e. goals) to which individuals feel committed (Fishbein \& Ajzen, 2009; Gallo, McCulloch, \& Gollwitzer, 2012), implementation intentions refer to the link between a specific cue and an intended behaviour or action. The approach is based on Heckhausen and Gollwitzer's (1987) ‘Rubicon’ Model of Action Phases which makes a distinction between motivational and volitional phases of action. Intentions are formed in advance of a volitional phase in which individuals prepare for action and it is this latter phase in which implementation intentions are critical. Implementation intentions make explicit the mechanisms that reduce the 'gap' between intentions and the attainment of a behavioural goal. These mechanisms are characterised by (1) an increased mental accessibility of a cue (or cues) to perform the behavioural response, obtained by specifying a situational cue (or cues) and (2) activating the behavioural response without conscious deliberation, in the presence of the cue(s) (Gallo et al., 2012; Orbell \& Verplanken, 2010; Webb \& Sheeran, 2004). Although forming an 'if-then' plan is a conscious act, the mechanism by which implementation intentions operate is hypothesised to be automatic and non-conscious rather than deliberative and conscious (Hagger \& Chatzisarantis, 2013; Strack \& Deutsch, 2004; Webb \& Sheeran, $2008)^{4}$.

Implementation intentions are typically operationalised as a link between situation and goal-oriented response (e.g., "If situation X is encountered, then I will perform response Y"), and therefore makes explicit the link between a highly-specific outcome after exposure to a

\footnotetext{
${ }^{4}$ It should be noted that the evidence for non-conscious and automatic processes has mainly been obtained in laboratory studies with simple behavioural responses measured immediately post-test or after a relatively short time gap. A few field trials have indicated that forming implementation intentions resulted in increased automaticity in the enactment of health behaviour. For example, Orbell and coworkers (1997) found that forming implementation intentions reduced the effects of past behaviour (a proxy for habit) on intentions for breast selfexamination and Orbell and Verplanken (2010) demonstrated that forming an implementation intention increased habitual, non-deliberate performance of dental flossing.
} 
highly specific cue or set of cues (Gollwitzer, 1999; Gollwitzer \& Sheeran, 2006). Implementation intentions do not make reference to the enactment of the target behaviour in other situations and deal with a single or narrow set of cues (e.g., "saying 'no, thanks' after being offered a favourite alcoholic drink"). Although Gollwitzer (1999) indicates that the situational cue accounts for 'when' and 'where' the behaviour will be enacted, implementation intention research in the field often adopts cues that do not necessarily accounting for actual time and location (e.g., "If I'm offered an alcoholic drink then ..."; Armitage, 2009). Gollwitzer (1999) stresses that the cues should be readily available in the environment and that they are not 'internal states'. In other words, the cue should not be one that is self-referenced (e.g., "When I am feeling angry I will....”). In laboratory-based research on implementation intentions the cue is often simple (e.g., "The letter 'Q"') and is provided by the experimenter. In field research, implementation intentions are typically administered as a condition in an intervention or experiment and are usually delivered in the form of a text-based exercise in which individuals are prompted to use the 'if-then' structure to write down a cue that will trigger the specified behaviour they plan to perform (Armitage, 2009; Orbell et al., 1997). The outcome of implementation intentions would be defined as the number of responses Y (saying 'no, thanks') after the individual was exposed to the cue X (an offer of a favourite alcoholic drink). It is important to note that Gollwitzer's original conceptualisation focused on developing a link between a cue, that is an unconditional event like a time of day or environmental event (e.g., "5pm on a working day"), with a narrowly-defined behavioural response (e.g., "go to the gym") which might be later followed by an uptake of the target behaviour (e.g., "participating in physical activity"). In this case, the behavioural measure that corresponds directly with the cue included in the implementation intention would be the frequency with which the planner picked up his or her gym bag and went to the gym. While this in itself may be a valuable behaviour change, researchers adopting implementation intentions in health contexts frequently make a direct link between the plan and an unconditional behavioural response measured as an index of a broad behavioural category (e.g., an increase in physical activity levels). While it is possible that an individual picking up his or her gym bag and going to the gym everyday on a working day may lead to an overall 
increase in physical activity levels, this would be an indirect effect as the behavioural measure (physical activity) does not correspond directly with the response specified in the individual's plan (Sniehotta, 2009). It has to be noted that, in the context of applied sciences, researchers tend to focus on the effects of implementation intentions on an unconditional behavioural response performed regardless of presence or absence of the cues included in the implementation intentions. Behavioural responses of interest are likely to be changes in healthrelated target behaviour, broadly defined, such as an increase in physical activity levels. This focus is due to differences in the ultimate goals of researchers in basic and applied sciences. Applied researchers focus on much broader behavioural outcomes as they are seeking for behaviour changes responsible for clinically significant health improvements. In contrast, basic researchers tend to focus on narrowly-defined behavioural responses that correspond closely with specified respective cues as their principal concern is developing an understanding of the precise mechanisms involved.

In contrast to implementation intentions, there appears to be no definitive definition of action planning in the literature. Implementation intentions have tended to be defined more clearly and there is a general consensus in the literature on exactly how this technique has been conceptualised and operationalised. This may stem from the fact that research on implementation intentions tends to follow the theoretical underpinnings of the Gollwitzer (1999; 2006) school and the 'Rubicon' model (Heckhausen \& Gollwitzer, 1987). Action planning, on the other hand, appears in numerous models such as the Health Action Process Approach (HAPA; Schwarzer \& Luszczynska, 2008), the Integrated-Change Model (I-Change Model; de Vries, Mesters, van de Steeg, \& Honing, 2005), the MoVo concept (Fuchs, Goehner, \& Seelig, 2011), and the Integrated Behavior Change Model (Hagger \& Chatzisarantis, 2013) and stems from relatively early in the social psychological literature (e.g., Leventhal et al., 1965). Generally, action planning often contains the same elements of implementation intentions, such as the cue-response contingency and the focus on a link being made between an unconditional cue and a behavioural response. In fact, the term action planning has been frequently used interchangeably with implementation intentions in the literature, and the two techniques are, in some cases, indistinguishable in content and focus. This has led researchers 
to allocate implementation intention and action planning interventions to the same category when synthesising effects of planning on health behaviour across the literature (Adriaanse, Vinkers, et al., 2011; Bélanger-Gravel et al., 2013).

However, there are also variations in the way in which action planning has been conceptualised and operationalised in the literature. These differences are centred about a broader perspective to the cue-to-action approach, the additional components that accompany action planning (e.g., specifying 'how' a behaviour is to be conducted, inclusion alongside coping planning), and the theoretical underpinning of the technique, such as those specified in the HAPA and the I-Change Model. Strictly speaking, the 'if-then' plans specified in implementation intentions tend to target a single cue-to-action response (Adriaanse, Vinkers, et al., 2011). The 'if-then' formula used in the implementation intentions studies is guided by standard methodological procedures, typical of laboratory research, aimed at evaluating immediate responses to exposure to a selected cue. In contrast, action planning tends to focus on a broader perspective, and it may include multiple cues and complex behavioural responses with respect to pursuing a goal intention (Luszczynska \& Schwarzer, 2003). The action planning approach, more frequently employed in field research, assumes that cues-to-action should make reference to time-related cues ('when') and the complex external environment ('where') (Luszczynska \& Schwarzer, 2003). Further, action planning often assumes the specification of 'how' the behaviour should be done. The 'how' component refers to the specific behaviour to be initiated on presentation of the stated cue (Schwarzer, 2008). The specified action may be simple (e.g., "running") or relatively complex in terms of the specific actions performed (e.g., "running for 30 minutes"). Finally, action planning is often seen as means to obtain a broader, more complex set of behavioural responses and less-specific behaviour change, such as an increase of physical activity, a reduction of fat intake, or the avoidance of unwanted pregnancy (Sniehotta, 2009). Besides forming action plans about when, where, and how to act, the action planning approach is generally accompanied by additional components aimed at narrowing the gap between plans and behavioural enactment such as the formation of coping plans (the anticipation of barriers and the generation of alternative behaviours to overcome them; Sniehotta, Scholz, Schwarzer, et al., 2005) or a requirement to 
self-evaluate the completeness of formulated plans and plan enactments (Bagozzi, Dholakia, \& Basuroy, 2003). The broader perspective and additional components like the specification of how the target action should be executed are consistent with alternatives to the Rubicon model (Heckhausen \& Gollwitzer, 1987).

The mechanisms involved in action planning may account for both non-conscious and conscious processes that have been proposed dual-systems models of action (Hagger, Anderson, Kyriakaki, \& Darkings, 2007; Keatley, Clarke, \& Hagger, 2012, 2013). The simple cue-to-action aspect of action plans may elicit behaviours automatically in a similar manner to implementation intentions (Schwarzer, 2008). However, forming action plans accounting for relevant time-related cues, the events in the external environment ('when' and 'where'), and a relatively complex sequence of actions ('how') requires effortful selection and self-evaluation processes (Bagozzi et al., 2003). The effects of 'when, where, and how' action plans on behaviour change are, therefore, likely to be mediated by conscious self-regulatory processes (Luszczynska, 2006). Further, including components such as coping plans alongside these plans implies a more effortful decision-making processes (Bagozzi et al., 2003). Action planning includes plan formation, self-evaluation of the completeness of formulated plans, and plan enactments (Bagozzi et al., 2003). Adjustments of plans in the form of coping plans (Sniehotta, Schwarzer, Scholz, \& Schuz, 2005), or repetitions of plans to overcome memory limitations and environmental changes (Luszczynska, 2006), all require deliberate, reasoned processing rather than non-conscious responses (Hofmann, Friese, \& Wiers, 2011; Strack \& Deutsch, 2004). In sum, one of the key differences between implementation intention and action/coping planning may be the involvement of deliberate, conscious processes of decisionmaking and self-evaluations, embedded in the formation of action and coping plans.

If the key factors involved in different planning strategies are to be isolated, it is important that the independent effects of implementation intentions and action plans on behaviour are elucidated. While the two approaches clearly share common elements, a fuller understanding of their effects would be gained from factorial research designs that compare the underlying mechanisms and their differential effects on behaviour. For example, we envision a study in which a 'pure' implementation intention condition, where participants specify a 
narrow 'if-then' plan (e.g., "If I see my sneakers in the morning, I will go for a run”), is evaluated against a condition in which participants augment their 'if-then' plan with a description of time, environment, and the description 'how' they will go about it (e.g., "I plan to do my running on Monday, Wednesday, and Friday at 6:30 am. I plan to do it in the West City Park. I plan to run for 30 minutes").

\section{Planning Approaches and Social-Cognitive Theories}

The recent increase in attention paid to implementation intention and action planning approaches in the health psychology literature may be due to a recognition of the need to address limitations of social-cognitive models that stress the central role of intention in explaining behaviour such as the theory of planned behaviour (Ajzen, 1985), protectionmotivation theory (Rogers, 1975), and the health belief model (Rosenstock, 1974). Metaanalyses of intention-behaviour relations have identified medium-to-large effect sizes in correlation-based analyses (Armitage \& Conner, 2001; Hagger, Chatzisarantis, \& Biddle, 2002; McEachan, Conner, Taylor, \& Lawton, 2012) but considerably weaker effects in experimental studies (Rhodes \& Dickau, 2012; Webb \& Sheeran, 2006). The intentionbehaviour 'gap' refers to the imperfect relation between these variables, particularly for experimentally-induced intentions (Armitage \& Conner, 2001; Hagger, 2010a; Hagger et al., 2002; Scholz et al., 2008; Sniehotta, Scholz, \& Schwarzer, 2005; Webb \& Sheeran, 2006). The 'gap' implies that substantial groups of individuals can be highly motivated and still not act upon their intentions, characterised as 'inclined abstainers' by Orbell and Sheeran (1998). A prominent explanation for why individuals fail to act on intentions is that their self-regulatory abilities are insufficient (Schwarzer, 2008). Such abilities may be manifested in the volitional components put forward in planning approaches and may serve to resolve the shortfall in the predictive capacity of social-cognitive theories (Bayer \& Gollwitzer, 2007; Brandstätter, Lengfelder, \& Gollwitzer, 2001; Gollwitzer \& Sheeran, 2006; Sheeran, Webb, \& Gollwitzer, 2005).

According to the 'Rubicon' model (Heckhausen \& Gollwitzer, 1987), on which implementation intentions are based, enactment of a behaviour has distinct motivational (or intentional) and volitional (or post-intentional) phases. Intentions are necessary but not 
sufficient to initiate and execute planned actions. Initiation of intended, goal-directed behaviour occurs in a post-intentional volitional phase and is facilitated by the presence of environmental cues which facilitate the recall and efficient implementation of intended acts (Heckhausen \& Gollwitzer, 1987). The identification of cues to prompt efficient enactment of behaviour therefore occurs after the decision or intention has been made to engage in the behaviour. Researchers have made explicit links between the processes outlined in the theory of planned behaviour, as the prototypical theory of intention, and the additional volitional components in the Rubicon model, and have tended to adopt the theory of planned behaviour (Ajzen, 1985) as a framework by which implementation intention approaches are operationalised (e.g., Orbell et al., 1997; Sheeran \& Orbell, 1999b; Sheeran \& Silverman, 2003). Importantly, measures of intention from the theory of planned behaviour have been found to remain unaffected by the formation of implementation intentions (Strack \& Deutsch, 2004; Webb \& Sheeran, 2008) providing confirmation that the planning strategy operates in the volitional phase and independent of intention.

Recognising the distinct phases proposed in the Rubicon model, researchers have suggested that interventions targeting both motivational and volitional phases may be most effective in promoting the initiation and uptake of a target behaviour (Hagger, 2009; Hagger, Lonsdale, Koka, et al., 2012; Milne et al., 2002). This has led to intervention designs that have adopted motivational components based on the social-cognitive models traditionally used to explain health behaviour alongside volitional components such as implementation intentions. The combined or interactive effect of the motivational and volitional components are expected to act synergistically and lead to greater behavioural engagement that either component alone. An intervention comprising solely of implementation intention would be limited as identifying cues to action would have little resonance without motivation to engage in the behaviour. Similarly, strategies to promote intention are often not converted into action due to extraneous circumstances or an inability to recall the intended action at the appropriate time and place (Sheeran \& Orbell, 1999a; Webb \& Sheeran, 2008). Recent evidence suggests that this is the case with more researchers adopting this synergistic approach to designing health-behaviour interventions that include both motivational and implemental components (Andersson \& Moss, 
2011; Chatzisarantis, Hagger, \& Wang, 2010; Hagger, Lonsdale, Koka, et al., 2012; Hagger, Lonsdale, \& Chatzisarantis, 2012; Knauper, Pillay, Lacaille, McCollam, \& Kelso, 2011; Knauper, Roseman, Johnson, \& Krantz, 2009; Milne et al., 2002; Prestwich et al., 2008; Prestwich et al., 2003).

The HAPA (Schwarzer \& Luszczynska, 2008) and I-change approaches (de Vries et al., 2003) represent a more elaborate integration of a number of traditional social cognitive models of intentional behaviour with planning as a key element. Action planning is an integral component of these models and, like the Rubicon model, they make a distinction between motivational and volitional phases and propose that planning will serve to account the shortfall in the link between intentions and behaviour across the phases. The action plans include cues to action ("when", "where"), identical to implementation intentions, but also steps to action ("how"). In addition, the models also specify another form of planning which operates in parallel to action planning, known as coping planning. Coping planning is more elaborate than the action plans and refer to plans to maintain the behaviour in the face of contingencies that arise during behavioural enactment such as barriers. In addition, the models also incorporate different forms of self-efficacy, namely, action self-efficacy, which reflects beliefs about ability to initiate and maintain in the target behaviour, and coping self-efficacy, which reflects beliefs about dealing with barriers and hurdles needed to overcome and maintain behaviour change. The effects of these forms of self-efficacy on intentions and behaviour are proposed to be mediated by action and coping planning, respectively. The models therefore do not hypothesise the proposed interactive effects between the motivational and volitional components. Instead, planning components serve as the mechanism by which intentions are enacted. Beliefs about ability to initiate given actions lead to the formation of planning which, in turn, leads to the behavioural enactment in the volitional phase. Formal tests of the HAPA and I-change models including the additional components of coping planning and forms of self-efficacy and using experimental and randomised trials are relatively rare (see Payaprom et al., and Teng, 2011 for examples), with much of the evidence for the elaborated model tested using correlational designs (e.g., Barg et al., 2012; Dohnke, Nowossadeck, \& Muller-Fahrnow, 2010). 
In summary, implementation intentions have been hypothesised as means to resolve the imperfect intention-behaviour relationship outlined in social cognitive models. This is a novel integration of the 'Rubicon' model and existing social cognitive theories of intention (e.g., the theory of planned behaviour) to resolve the intention-behaviour 'gap' in the existing theories. The HAPA and I-change approaches can be seen as emerging from this novel application and other social-cognitive traditions, but propose more complex explanations through elaborated plans (e.g., including coping planning) and, most importantly, embedding these plans in an integrated model that incorporates constructs from other social cognitive models such as the theory of planned behaviour and social cognitive theory. The distinction between intentional and volitional phases in these models suggest that hybrid interventions involving both motivational and planning components offers considerable promise for behaviour change interventions. As the literature expands and more tests of these integrated approaches are conducted, a synthesis of the effectiveness of these interventions compared to singlecomponent interventions (e.g., implementation intention only) is warranted. Studies that include the additional component of coping planning and forms of self-efficacy in experimental studies and randomised trials are needed to elucidate the role that these components may play, particularly alongside the effects of hybrid motivational and planning designs on health behaviour.

\section{Format and Measurement}

Although laboratory studies testing the effectiveness of implementation intentions and action planning tend to adopt a similar format when presenting the techniques (Bayer \& Gollwitzer, 2007; Brandstätter et al., 2001; Gollwitzer \& Sheeran, 2006; Sheeran, Webb, et al., $2005)$ there is considerable variation in the procedures used to deliver implementation intention or action planning protocols in the field studies. The procedural differences may affect the size and sustainability of the effects of the planning interventions on behavioural outcomes. The procedural differences in terms of the characteristics and protocol of these interventions impose limitations on attempts to generalise results of planning interventions across the literature. In this section, we identify and discuss these procedural variations, offer insight into how they 
likely influence the effectiveness of planning interventions, and outline some recommendations as to how researchers may iron out these variations.

\section{Mode of Delivery}

A key difference in planning intervention protocols is the mode of delivery. Orbell et al. (1997) set a precedence in the delivery of implementation intention manipulations in health contexts by embedding them within questionnaires using a pen-and-paper exercise with prompts to write down the 'when' and 'where' components of the cues to action. Since then, this format has been the dominant approach in studies adopting planning interventions in health contexts, mainly because of the relative ease of delivery and the potential applicability of such a format to real-world scenarios (e.g., leaflets, pamphlets, letters etc., Abraham, Southby, Quandte, Krahe, \& van der Sluijs, 2007). A similar approach has also been taken in electronic and internet-based interventions using implementation intention components in which participants are prompted to type rather than write the cues to action (e.g., Craciun, Schuz, Lippke, \& Schwarzer, 2012; Hagger, Lonsdale, \& Chatzisarantis, 2012; Tam, Bagozzi, \& Spanjol, 2010). One of the advantages offered by this approach is that it minimises any method variance that could potentially be introduced due to the presence of a researcher, facilitator, or practitioner thereby isolating the effect of the intervention components alone.

In contrast, some researchers have delivered the planning interventions in one-on-one interview sessions with participants prompted to form 'if-then' plans by a facilitator and given follow-up feedback on plan formation and a reward for constructing specific and high quality plans (Luszczynska, 2006; Luszczynska, Sobczyk, \& Abraham, 2007). Such methods augment the typical prompts to form 'if-then' plans with social support and incentives, and allows for the development of more elaborate plans due to personalised feedback. These protocols are more in keeping with the action planning approach which incorporate more than mere specification of cues to action, but also include means to overcome barriers and means to cope with contingencies. Such delivery has the disadvantage of the presence of a facilitator to administer the intervention components which may interact with the content. Such interactive effects have not been formally tested and we look to future research to establish the 
comparative effectiveness of these delivery approaches and relate them to the intervention components alone.

\section{Measurement Effects}

Some conceptualisations of planning do not require participants to actively engage in the types of pen-and-paper exercises like those typically used in implementation intentions, instead they merely ask participants to report the extent to which they have formed plans (Churchill \& Jessop, 2010; Conner et al., 2010; Godin, Sheeran, et al., 2010). These types of measures may also be used in implementation intention studies to ascertain the fidelity of the intervention in terms of whether participants engaged in the implementation intention exercises. Recent research seems to imply that the mere fact of measuring planning may serve as a manipulation in itself (Conner et al., 2010; Godin, Sheeran, et al., 2010). These findings are consistent with research that has demonstrated 'mere-measurement' effects of socialcognitive measures on actual behaviour (Godin, Sheeran, Conner, \& Germain, 2008; O'Sullivan, Orbell, Rakow, \& Parker, 2004; Sandberg \& Conner, 2009). Although there is considerable heterogeneity in the size and extent of mere-measurement in the health psychology literature, the possibility raises questions regarding the format and nature of planning measures and the role that fidelity checks may have on the behaviour of control or comparison group participants. It is possible that planning measures may not necessarily require elaborate exercises to bring about short-term behaviour change. Furthermore, interventions involving measures of planning to evaluate the extent to which participants engage in planning exercises may need to establish whether the measures may affect change in both the intervention and control groups. An option that may help researchers resolve these effects is to adopt a Solomon (1949) four-group design, which allows researchers to establish the independent and interactive effects of measures and manipulations in one experimental design. This would provide important evidence as to the nature of the effects of planning measures on behaviours and how such measures may interact with planning techniques.

\section{Format}

Researchers have also started to augment or manipulate the format of the written form of planning components. Such modifications include the format of the planning intervention, 
the provision of examples of plans (Luszczynska et al., 2007; Webb, Sheeran, \& Luszczynska, 2009), the use of externally- or user-defined plan formats (Armitage, 2009), and the use of individual and dyadic implementation intentions (Burkert, Knoll, Luszczynska, \& Gralla, 2012; Prestwich et al., 2005; Prestwich et al., 2012). In manipulating the format of the planning intervention, researchers seek to establish the most effective and parsimonious means to encourage people to identify relevant cues-to-action and enhance the strength of the link between cue and action. The varied format components are based on the Rubicon model hypothesis that identifying the appropriate cue will enhance recall of the intended action. In addition, format variations such as dyadic or collaborative implementation intentions employ other social processes that may augment the strength of the link between the cue and the action. Although some researchers have provided exact details of the planning interventions used in their trials, such as Armitage et al.'s (Arden \& Armitage, 2012; Armitage, 2008; Armitage \& Arden, 2010) volitional help sheet, complete protocols are rarely made available. The lack of clear reporting makes it difficult to establish the precise components of a planning intervention and evaluate the extent to which the intervention complies with theoretical specifications and those conducted by other researchers. This presents a challenge to researchers wishing to replicate the study protocol and undermines efforts to evaluate the cumulative effects of replications of planning interventions in the literature (Michie \& Abraham, 2008). In the next sections we outline the merits of format manipulations used in conjunction with the planning techniques in health behaviour research and identify important outstanding issues and gaps in the literature.

Global vs. 'if-then' plans. Traditional text-based planning intervention prompts typically require participants to write down (or type) a 'global' plan in a free-response format (e.g., Armitage, 2004; Jackson et al., 2005; Sheeran \& Orbell, 2000), which deviates somewhat from the requirement that the cue or 'critical situation' is linked with the action. This is problematic as the crux of the mechanism for the effect of implementation intention on behaviour is to increase the accessibility and salience of the cue as a prompt to action. Recently, Chapman et al. (2009) have formally compared the 'open ended' or global approach with a more 'closed' approach where participants are forced to specify their plans in an 'if- 
then' format advocated by Gollwitzer (1999). The 'if-then' format was found to be superior in producing behaviour change in fruit and vegetable intake. By explicitly requiring the formation of plans in the 'if-then' format, the link between the critical situation and action was reinforced and lead to more effective behavioural engagement in keeping with theory.

Examples. Researchers have also provided examples of 'if-then' plans alongside the prompts to form cues to action to assist individuals in developing their own plans (Chapman \& Armitage, 2010). Including examples may prime the respondent to select appropriate and specific cues or responses in their own plans, which may otherwise be poorly suited to participant's goals, environment, and past experience with the behaviour. Omitting an example may also affect participants' understanding of the prompt and lead them to form inappropriate or irrelevant cues. Some implementation intention interventions include examples of cues to action based on elicitation studies and therefore guide the formation of appropriate cues, which may not be immediately apparent to the person (Luszczynska et al., 2007; Webb et al., 2009). Participants may also be presented with a check list offering a number of examples of cues to action and asked to identify personally-feasible cues from the list (Conner \& Higgins, 2010). Such a check-list may be followed by an option for the individual to provide their own cue if those proposed on the list are unsuitable or omit cues which may not be personally relevant (Conner \& Higgins, 2010). Another recent approach is to to provide comprehensive lists covering the majority of critical cues and possible behavioural responses such as Armitage's (2008) volitional help sheet. Use of a structured volitional help sheet, consisting of multiple critical situations (cues) and behavioural responses, has been shown to be effective in prompting changes in a number of health behaviours including smoking cessation (Armitage, 2008), physical activity uptake (Armitage \& Arden, 2010), and alcohol reduction (Arden \& Armitage, 2012).

The use of examples does, however, need to be handled with care and qualified by appropriate instruction so that participants do not feel 'straitjacketed' in forming plans which are similar or identical to the examples provided. The latter may be detrimental to the mission of the implementation intention technique which aims to develop critical cues that are relevant and salient to the individual. To date, we know of no research that has formally ascertained 
whether the provision of examples enhances the implementation intention effect alongside more traditional forms of implementation intentions or leads participants to form more effective, or even, more confined implementation intentions; this is clearly an area that needs greater input.

Self vs. other-defined plans. The role of examples also gives rise to another important aspect of implementation intentions and action plans; the role of personal input or selfdefinition of plans. From the previous discussion, it would appear that leaving individuals to their own devices through global or open ended implementation intention manipulations may lead to the specification of inappropriate cues to action. This has been supported by research revealing that when given minimal guidance individuals tend not to develop high quality plans in terms of the appropriate cues and actions required to bring about a distal behaviour (e.g., condom use), but those who were prompted to form implementation intentions for preparatory behaviours that would bring about the distal behaviour (e.g., buying, carrying, and discussing condom) formed more appropriate, high-quality plans (de Vet et al., 2011). However, a key issue within Heckhausen and Gollwitzer's (1987) theory is the importance of self-relevant cues to action enhance the efficient recall and enactment of intended actions. This has been formally tested in the implementation intention literature. There is evidence that framing implementation intentions in terms of personally-relevant goals appears to be more effective in leading to better behavioural engagement (Adriaanse et al., 2009). There is, however, research suggesting that there is no difference in the effectiveness of user-defined and researcher-defined implementation intentions on behavioural engagement (Armitage, 2009). Examining the content of the different implementation intention manipulations in these studies suggests that provided the cues are relevant, the source of the plans, whether externally imposed, such as by an experimenter, or internally specified by the user themselves, is less relevant.

An interesting addition to this literature is evidence that planning in conjunction with others might be more effective than planning alone (Burkert et al., 2012; Prestwich et al., 2005; Prestwich et al., 2012). It has to be noted, however, that the effects of individual and dyadic planning on behaviour may be similarly negligible on long term follow-ups (Burkert et al., 2012). The mechanisms behind the effects of dyadic planning are unclear given that it is the 
strength of the link between the critical cue and action rather than the perceived beliefs of others or perceived social support that is the mechanism for the implementation intention approach (Prestwich et al., 2012). One possibility is that the collaborator may be a more salient, and, therefore, more accessible, prompt to act than other environmental cues, although this has yet to be tested. It is also possible that dyadic planning operates indirectly, via complex social-exchange mechanisms, accounting for the interplay between social control and received support (Burkert et al., 2012; Burkert, Scholz, Gralla, \& Knoll, 2011). Together the research on the format of planning interventions suggests that provided the cues are salient to the individual, the source of the cue to action, external or internal is less relevant, although it seems that forming the implementation intention or action plan in conjunction with others may lead to more effective behavioural engagement. There needs to be more input into the mechanism behind the collaborative effect.

Format is clearly an important aspect of any planning intervention, and research on format variations in planning intervention has demonstrated that it may have a pervasive effect on intervention effectiveness. Drawing from this research, it seems that encouraging individuals to form 'if-then', rather than global, plans with salient cues is important. Future research needs to disentangle the level of the specificity of the cues included in the plans from the format of plans. Open formats for plans based on self-generated cues may increase the likelihood that individuals will generate cues that are less specific and more vague (e.g., "if somebody offers me an unhealthy snack...", compared to "if my friend offers me chocolate..."). Whether the cues are imposed or self-generated has yet to be fully resolved, although there is preliminary evidence that forming implementation intentions or action plans in collaboration with significant others may facilitate their effectiveness.

\section{Mechanisms and Moderators}

\section{Habits and Planning Interventions}

The fact that many health-compromising behaviours tend to enacted with little conscious thought (e.g., smoking, snacking, drinking alcohol) and are the result of long-term repetition and reinforcement of behavioural responses to situational cues presents a serious problem to health professionals attempting to change behaviour in the face of such strong 
impulse-linked habits (Orbell \& Verplanken, 2010). One of the appealing assumptions of planning techniques is that they may help break habitual responses. Laboratory-based research has suggested that plans render the habitual response less accessible relative to the counterhabitual implemental response (Adriaanse, Gollwitzer, De Ridder, de Wit, \& Kroese, 2011; Webb et al., 2009). Adriaanse et al. use a 'horse-race' metaphor to explain this phenomenon, suggesting that the formation of an implementation intention leads to a strengthening of the link between the specified cue and counter-habitual action and an inhibition of the link between the cue and the habitual response. This leads to the cue-action link specified in the implementation intention to 'win' in the 'horse race' with the cue-habitual response link. The researchers demonstrated empirically that the accessibility of the habitual action was inhibited among those forming implementation intentions compared to those who did not form implementation intentions. Forming 'if-then' plans may, therefore, be an effective mean to break habitual responses (or form new habits) (Fleig et al., 2013; Hagger, 2010b; Hagger, Wood, Stiff, \& Chatzisarantis, 2010; Webb et al., 2009).

However, the effectiveness of planning interventions in overcoming habitual responses may be moderated by a number of factors such as the strength of the habit and type of behaviour (e.g., approaching a healthy behaviour vs. avoiding an unhealthy one). One of the defining characteristics of habits is that they are developed over a considerable length of time allowing for long-term reinforcement of cue-response linkages. On the other hand, forming implementation intentions usually involves rehearsing cue-response links a small number of times. Thus, the new cue-response link is considerably less frequently rehearsed than that of the habitual response and has therefore not received the same level of reinforcement.

Differences in the extent of rehearsal and reinforcement may favour stronger situation-response links (such as well-ingrained habits) compared to the behavioural alternative prompted by planning interventions. For example, some behaviours (e.g., eating snack foods) have multiple environmental and internal cues linked to the same unwanted response (e.g., sadness, being bored, drinking coffee, watching TV, meeting up with a friend). Furthermore, such behaviours may have been reinforced on multiple occasions by means of a reduction of a variety of unpleasant emotional or physical states (e.g., a reduction of tension or enhanced concentration 
after smoking a cigarette). It is important to note that the stronger an individual's habit the less effective implementation intentions will be. Research has shown that the effect of implementation intentions on behavioural engagement is stronger for those with weaker habits compared to those with stronger habits (Adriaanse, van Oosten, de Ridder, de Wit, \& Evers, 2011; Webb et al., 2009). Interestingly, research has shown having a strong goal intention for a counter-habitual action is important for implementation intentions to be effective in breaking habits. Therefore, it may be that augmenting planning interventions with components to foster goal intentions for alternative actions among individuals with strong habits will increase the effectiveness of implementation intentions to break habits (Adriaanse, Gollwitzer, et al., 2011).

Interestingly, recent research suggests that the effectiveness of planning interventions in breaking habitual response may vary if the 'if-then' plan specifies a behavioural response that is akin to avoiding the unwanted behaviour rather than approaching an adaptive behavioural alternative. For example, Adriaanse et al. (2011) found that specifying an implementation intention to avoid an unwanted behaviour (e.g., "If I feel sad then I will not eat chocolate") was less effective in changing the desired behaviour of avoiding snack foods than an implementation intention that made reference to a desirable alternative or replacement behaviour (e.g., "If I feel sad I will eat an apple"). In fact, the former 'negating' implementation intention led to the ironic effect of increasing accessibility of the unwanted behaviour and snack food consumption (cf., Wegner, Schneider, Carter, \& White, 1987). Consistent with these findings, a recent systematic review indicates that approach-oriented implementation intention or action planning interventions result in larger effects on behaviour than avoidance-oriented planning interventions (Adriaanse, Vinkers, et al., 2011). It is not known, however, whether the difference in effects stems from the use of approach or avoidance implementation intention or action planning interventions or from the fact that it is easier to create a new habit (when there was a lack of a competing behavioural habit at the baseline) than counteract strong behavioural habits (habit present at the baseline). This represents a clear avenue for future research to establish whether negating or approach implementation intentions are more effective in overcoming behaviours that vary in habit strength. 
Finally, the 'regulatory fit' of the planning approach may be an important moderating factor when it comes to the effectiveness of implementation intentions in overcoming habits. In line with the ecological rationality rule (Kruglanski \& Gigerenzer, 2011), selecting implementation intentions with better fit to the individual's internal and external environment is likely to lead to more effective behavioural enactment. Fitting implementation intention interventions with the self-regulatory preferences of the individual may be helpful in breaking stronger habits. Research has shown that individuals with weak unhealthy snacking habits benefited from forming any plans and reported eating fewer snacks, whereas individuals with stronger snacking habits reduced snacking behaviour only when the specification of the implementation intention (approach vs. avoidance) exhibited good-fit with their self-regulatory focus, defined as a preference to use strategies aiming at prevention or promotion (Tam et al., 2010). Researchers should therefore be sensitive to the preferences of the target population, in terms of approach or avoidance of unwanted behaviours and their habit strength, when developing planning interventions that are effective in overturning ingrained habitual healthrelated behaviours.

\section{Moderators}

As with any theory-based intervention technique, health psychologists are interested in generalisability of planning techniques on behavioural outcomes across contexts and behaviours. This is an important endeavour as it will provide an indication as to whether the adoption of these techniques will lead to overall behaviour change at the population level. Verification of the generalisability necessitates research to demonstrate the efficacy of the planning techniques in changing behaviour across numerous behavioural domains and populations, but also the evaluation of the potential moderators of the effect. This will provide an indication of not only the most effective strategies and operationalisation of planning interventions for most efficient and effective behaviour change, but also enable the identification of the contexts and factors that need to be taken into account when designing interventions using these techniques. We have already identified habit strength as a key individual difference variable that affects the effectiveness of these planning approaches in the previous section. Research has shown that planning interventions have been moderated by a 
number of factors including personality factors, executive functioning, social environment, and macro-level environmental variables. We provide a brief overview of each of these factors in this section with a view to identifying the important contribution that individual difference variables have made to understanding the mechanisms underpinning planning interventions and also the important gaps in the literature that need to be addressed in future research.

Intention strength. Intention strength ${ }^{5}$ has been identified as a moderator of the effects of implementation intentions and action planning on behaviour. The Rubicon model assumes that the formation of intentions is a prerequisite for the enactment of a given future behaviour (Heckhausen \& Gollwitzer, 1987). Strong intentions are considered an essential condition for entering into the volitional stage to act (Brandstätter et al., 2001; Heckhausen \& Gollwitzer, 1987) and they have been shown to moderate the effects of volitional interventions such as implementation intentions on behaviour (Sheeran, Webb, et al., 2005). For example, experimental research has demonstrated that the effectiveness of implementation intentions is dependent on the strength of intentions with greater goal attainment reported among participants who formed implementation intentions and had stronger goal intentions (Sheeran, Webb, et al., 2005). Unsurprisingly, most planning interventions have the formation of an intention to act as a prerequisite with the implication that the stronger the intention the more effective planning manipulations will be in promoting behavioural engagement.

In contrast, there is evidence suggesting that implementation intentions are more effective among individuals with weak intentions (Godin, Belanger-Gravel, Amireault, Gallani, et al., 2010). A possible reason for these conflicting results may be the context (laboratory vs. field) and the outcome (behavioural engagement vs. goal attainment). There is a need for further research examining this interaction effect, particularly laboratory and field experiments that manipulate both planning intervention and intention strength. This will have implications for the discussion of hybrid interventions to promote health behaviour involving motivational (intention-forming) and volitional (planning) components. While intention may be a

\footnotetext{
${ }^{5}$ Intention strength has generally been conceptualized as the level or magnitude of self-reported intentions, that is, how high one rates his or her intention on psychometric items measuring the construct. It has, however, also been defined as the temporal stability of intentions, that is, the extent to which the construct varies over time. In the current view, we refer to intention strength in terms of its level or magnitude.
} 
prerequisite for the effectiveness of a planning intervention, it remains to be seen whether plans will be more effective in facilitating behavioural enactment among those with stronger or weaker intentions. This may determine the nature of the motivational component of hybrid interventions.

Self-efficacy. Self-efficacy is frequently-measured alongside planning intervention components in research on health-related behaviours. In research comparing the effects of selfefficacy against planning strategies, research has suggested that planning may be more effective than fostering self-efficacy beliefs in the prediction of behaviour. For example, research has shown that implementation intention interventions are more effective in promoting health behaviour than self-efficacy interventions in the context of smoking cessation (Conner \& Higgins, 2010) and fruit and vegetable intake (Guillaumie, Godin, Manderscheid, Spitz, \& Muller, 2012). In terms of mechanisms, self-efficacy has been conceptualised as a moderator of the effects of action planning on behaviour. For example, there is research to support an interaction between action plans and self-efficacy on behaviour suggesting that selfefficacy may facilitate engaging in action planning activities and thus prompt more effective behaviour change (Bayer \& Gollwitzer, 2007; Luszczynska et al., 2010; Luszczynska, Schwarzer, Lippke, \& Mazurkiewicz, 2011). In addition, the beneficial effects of implementation intentions on the performance of complex and demanding tasks has been shown to be dependent on efficacy beliefs, with strong self-efficacy a prerequisite for obtaining significant effects of a planning intervention on goal attainment (Wieber et al., 2010). However, evidence for the interactive effects of self-efficacy and planning intervention on health behaviour is relatively scarce, although research measuring self-efficacy and related constructs from social-cognitive models (such as perceived behavioural control from the theory of planned behaviour) have demonstrated no effects of planning manipulations on self-efficacy (Arbour \& Martin Ginis, 2009; Barg et al., 2012; Conner et al., 2010; Gellert et al., 2012; Luszczynska, 2006; Milne et al., 2002; Prestwich et al., 2012; Prestwich et al., 2003). This is in keeping with Heckhausen and Gollwitzer's (1987) Rubicon model in that planning is supposed to occur in a post-decisional manner and therefore have no affect on the variables linked to decision making like self-efficacy. 
It is, however, important to note that much of the research examining the interactive effects of self-efficacy and planning interventions on behaviour have tended to adhere to a definition of self-efficacy as action self-efficacy (beliefs in ability to execute the behaviour in the future), which are likely to be formed in the motivational phase according to models such as the HAPA and I-Change. Other forms of self-efficacy such as coping self-efficacy, which involves the ability to manage the behaviour in the face of barriers and challenges, tends to operate in a post-decisional manner (Ochsner, Scholz, \& Hornung, 2013). Coping self-efficacy may also operate as the mediator between the first attempts to act upon formed plans (or undertaking preparatory behaviours) and the maintenance of a target behaviour (Koring et al., 2013). In models like the HAPA such self-efficacy perceptions are proposed to mediate the effects of planning on behaviour, although there are few experimental tests of this hypothesis. In summary, there is evidence to suggest that efficacy beliefs may moderate the effect of planning interventions on behavioural enactment, although planning does not seem to have an effect on self-efficacy per se. Future research may need to investigate if the association between planning and self-efficacy is mediated by efficacy beliefs specifically referring to ability to form plans rather than beliefs about ability to initiate or maintain healthy behaviour.

Impulsivity and executive functioning. There is evidence that individual difference variables that reflect the tendency to engage in behaviours spontaneously according to impulses and urges with little reflective, deliberative processing may undermine the effectiveness of planning interventions. Research has demonstrated that trait impulsivity, that reflects the propensity of an individual to succumb to impulse-related behavioural stimuli, moderates the effectiveness of action planning on health behaviour (Churchill \& Jessop, 2010; Churchill \& Jessop, 2011). Accounting for impulsivity may help to explain the relation between planning intervention components and habit strength. Strong habits, compulsive behaviours, and impulsivity share neurobiological underpinnings and are characterised by reduced activity in the orbitofrontal cortex, the brain region associated with impulse control and inhibition of welllearned prepotent responses (Torregrossa, Quinn, \& Taylor, 2008). Therefore high impulsivity may negate the impact of planning interventions, perhaps by inhibiting the capacity of the new cue-action response link specified in the implementation intention in favour of the more well- 
learned, habitual cue-action response link. The habitual route to action may, therefore, be more likely to 'win out' consistent with Adriaanse et al.'s (2011) 'horse race' analogy. However, the role of impulsivity needs to be further investigated as the mechanisms have yet to be established empirically.

Linked to impulsivity, limitations in executive functioning may also interfere with the self-regulation of action and attaining difficult, distal goals. Executive functioning reflects an individual's capacity for attention, planning, response inhibition, and action monitoring. It therefore stands to reason that some of these functions are likely to be involved in an individual's ability to engage in goal-directed behaviour (Hagger \& Chatzisarantis, 2013; Hagger, Keatley, \& Chan, in press; Hall \& Fong, 2010). Evidence suggests that impairments in executive functioning are associated with low intention-behaviour relationships, likely because such individuals have an impaired capacity to engage in complex, long-term planning (Hall, Fong, Epp, \& Elias, 2008; Mullan, Wong, Allom, \& Pack, 2011). Planning manipulations like implementation intentions as simple regulatory strategies may be useful in minimising the demands of difficult tasks on executive function and thus improve behavioural enactment among those with lower executive functioning. Experimental studies have revealed that implementation intentions improved performance on executive functioning in children with ADHD (Gawrilow, Gollwitzer, \& Oettingen, 2011b) and on delay of gratification tasks in children with and without ADHD (Gawrilow, Gollwitzer, \& Oettingen, 2011a). Field studies further highlight the role of implementation intentions among individuals with lower executive functioning, performing their physical activity under challenging environmental conditions (Hall, Zehr, Ng, \& Zanna, 2012). If the environmental conditions are challenging (cold weather, high precipitation) the formation of planning components like implementation intentions results in goal attainment, but this effect is most marked among individuals with weaker executive functioning. Further, implementation intention interventions are effective in promoting simple goals among individuals who score low on conscientiousness, compared to those who report high levels of self-organisation and deliberation (Webb, Christian, \& Armitage, 2007). Executive functioning appears to be an important moderator of planning interventions on health behaviour and, alongside impulsivity, a variable that likely reflects 
limitations in executive functioning, should be taken into account when designing interventions adopting planning interventions.

Cue accessibility. Effectiveness of planning interventions depends on accessibility of the cues specified in planning intervention to the individual. There is considerable evidence that the accessibility of salient social-cognitive constructs is a key moderator of their predictive validity (Doll \& Ajzen, 1992; Verplanken, Hofstee, \& Janssen, 1998), and the same process is proposed to operate within the Rubicon model with respect to planning interventions. Plans are more likely to prompt behaviour change if the specified cues are easily accessible to the individual. This means that the cues not only have to be relevant to the individual and appropriate to the target behaviour, but should also be readily accessible and have a strong association with the intended behaviour to activate the intended plan to act. This is opposed to obscure, non-salient, inappropriate cues with a weak link with the intended behaviour which will be less effective in triggering a plan of action. Experimental research has demonstrated that accessibility of the cue and strength of the cue-response association, measured by response latencies to primed versus neutral cues in a sequential priming task, mediate the effect of implementation intentions on behavioural responses (Webb \& Sheeran, 2008). Thus a key mechanism for the effectiveness of implementation intentions is cue accessibility, and in the absence of accessible cues, the individual may rely on more accessible alternatives such as a habitual action. This relates back to the 'horse race' model proposed by Adriaanse et al. (2011). In the absence of a readily accessible alternative, an individual is likely to fall back on welllearned associative responses, such as those formed by habit.

\section{Multiple and Repeated Plans}

According to the evidence discussed thus far, forming an appropriate, accessible, behaviourally-relevant plan is likely to be most effective in promoting behavioural engagement. However, as with many behaviour-change techniques, the formation of plans and subsequent execution of the intended behaviour do not occur in a social vacuum and one of the reasons why the effect sizes of laboratory versus field experiments evaluating the effectiveness of planning interventions may vary is that the advent of additional information or changes in environmental circumstances interfere with the cue-to-action linkage or provide alternative, 
highly-accessible courses of action that did not exist when the original plan was formed. Furthermore, the planning process tends to be more complex than previously thought in that individuals tend to form multiple plans that are contingent on numerous internal and external events. This has led researchers to investigate the role that the formation of multiple plans has in assisting individuals to deal with circumstances that may derail or interfere with their original or preferred plan. A related strategy is the role of repeated plans in which individuals are prompted to undergo the planning process on multiple occasions, whether or not the plans vary in content (i.e., are multiple) or remain the same. There are three likely approaches. First, an intervention may prompt the formation of multiple plans in one session, using an 'if-then' format, and sharing a common flat and non-ordered structure. Second, an intervention may be designed to assist the formation of a more organised structure of plans, with a sequence of simple 'if-then' plans followed by plans designed to cope with a change in environmental circumstances or events (e.g., coping plans; Sniehotta, Schwarzer, et al., 2005), or plans for behaviour initiation, maintenance, and relapse prevention (e.g., de Vries et al., 2003). Finally, the intervention may prompt the formation of plans on more than one occasion, and this may involve additional plans or a repetition of previously-made plans in order to reinforce them.

The original conceptualisation of the implementation intention approach assumed the formation of a single 'if-then' plan to link the intended behavioural response to a cue and has been verified as an effective strategy in laboratory-based studies (e.g., Adriaanse, Gollwitzer, et al., 2011; Brandstätter et al., 2001; Webb \& Sheeran, 2008) and field studies (e.g., Sheeran, Webb, et al., 2005). Recently, research has examined the effects of forming multiple plans including two (Hagger, Lonsdale, \& Chatzisarantis, 2012), three (Tam et al., 2010; Wiedemann, Lippke, Reuter, Ziegelmann, \& Schuz, 2011), or even five (Prestwich et al., 2012; Wiedemann, Lippke, \& Schwarzer, 2012) 'if-then’ plans. In fact, the number of plans has been shown to positively predict health behaviour engagement, with five plans leading to the greatest engagement (Wiedemann et al., 2012). Interestingly, it seems that, unless constrained, individuals will also make more than one plan. For example, when not prompted to make a specific number of plans, overweight or obese individuals made an average of 2.18 plans with respect to physical activity participation (De Vet, Oenema, Sheeran, \& Brug, 2009). The 
positive effect of forming more than one self-generated plan on physical activity was found only if the additional plans were specific (De Vet et al., 2009). Forming multiple plans may help individuals manage changes in their environment that may hijack the cue-action link, such as if a cue fails or ceases to appear. However, forming multiple plans needs to be handled with care as they may be detrimental to the enactment of plans and have the opposite effect by making decision making more effortful and demanding. Multiple plans may tax self-regulatory resources during implementation intention formation or activate competing goals and therefore inhibit reactions to cues (Hagger, Wood, Stiff, \& Chatzisarantis, 2009).

Recognising that individuals likely develop multiple plans to manage their behaviour (De Vet et al., 2009), researchers have developed planning protocols that prompt individuals to select a series of cues tied to a number of behavioural responses. In the context of the HAPA and I-Change models, a sequence of 'if-then' plans accompanied by coping plans to manage potential derailing contingencies (e.g., "if... [the obstacle] arises... then I will... [reaction]") has been proposed (de Vries et al., 2003; Sniehotta, Scholz, \& Schwarzer, 2006; Sniehotta, Schwarzer, et al., 2005). The situational cues used to manage potential barriers may be divided into merely observing tempting stimulus (e.g., "smelling pastry") versus noticing a lapse (e.g., “taking a bite"), with different responses (e.g., "diverting attention" or "saying to myself 'don't do it"') (Quinn, Pascoe, Wood, \& Neal, 2010). Such contingencies may be especially pertinent in health contexts as relapse prevention is a frequent target of health-related interventions (Luszczynska et al., 2011).

Multiple plans may be formed in one session (De Vet et al., 2009) or a formation of a plan may be repeated over several intervention sessions (Chapman \& Armitage, 2010). For example, compared to a single implementation intention, adding 'booster' implementation intention sessions with self-generated plans has been shown to increase adherence to fruit and vegetable consumption (Chapman \& Armitage, 2010). Seven repetitions of planning sessions over a period of two years affected adolescent smoking at long-term follow-ups (Conner \& Higgins, 2010). These 'booster' planning sessions allow participants to either repeat the original plans or come up with another plan that is a more suitable alternative. Repeated planning formation across several intervention sessions may help to overcome issues related to 
environmental changes and link different cues to the same behavioural response (Koestner et al., 2006). Such benefits may occur if different plans are formed in consecutive sessions. On the other hand, individuals may replicate their original plans and repeat them across the intervention sessions. This strategy would limit the vulnerability of planning interventions to memory decay and cognitive interference over time (Koestner et al., 2006). The boosters serve to enhance the recall of the cue to action which should, in turn, assist the recall of the linked intended action. Unfortunately, there are no formal tests of whether repeating the same plans across several sessions or forming different plans over the booster sessions is more effective. Repeated planning sessions may offer an important advance in the management and delivery of planning interventions. The provision of boosters, like the initial planning intervention, is relatively cost-effective with minimal intrusiveness and response burden. Furthermore, boosters can be delivered using novel and innovative means such as text messaging which can reach a large audience with few cost implications (Prestwich, Perugini, \& Hurling, 2009; Schwerdtfeger, Schmitz, \& Warken, 2012).

Nevertheless, there are some issues that need to be resolved in the contexts of multiple plans. It is currently unclear whether the effects of multiple plans lead to possible conflicts in terms of actions and behaviours. If this is the case, it may make it necessary for researchers to impose the plans on the participants or, at least, to guide the selection of the cues and plans. Future research should test for the differential effectiveness in planning interventions that prompt selection of multiple cues compared to single cues, as well as planning interventions that prompt selection of multiple behavioral responses compared to single responses. Further, while the evidence is encouraging for the greater effectiveness of combining action and coping action planning on behavioural enactment in health contexts, compared to forming simple action plans (de Vries et al., 2003; Sniehotta et al., 2006; Sniehotta, Schwarzer, et al., 2005), the mechanisms remain unclear. Such multiple plans may increase the flexibility of the individual with respect to their behavioural engagement in that they have several additional alternatives available to cope with contingencies. This has advantages over the single-plan approach as individuals will not have 'all their eggs in one basket' when it comes to cues to action. However, this has yet to be elucidated empirically, and research is needed to identify 
the mediators of the greater effectiveness of augmented planning programs that included 'ifthen' plans alongside more elaborate action and coping planning interventions.

\section{Planning and 'Low Intenders'}

Models such as the Rubicon and HAPA assume that forming an intention precedes plan formation and, therefore, assume that planning interventions are unlikely to be effective among those with low or no intentions to engage in the target behaviour. As we previously mentioned, research that has shown reduced effectiveness of implementation intentions among those with low intentions (Godin, Belanger-Gravel, Amireault, Gallani, et al., 2010; Guillaumie et al., 2012; Sheeran, Webb, et al., 2005) leading to a trend toward synergistic intervention designs incorporating motivational intervention components alongside planning interventions (e.g., Hagger, Lonsdale, Koka, et al., 2012; Milne et al., 2002; Prestwich et al., 2008). The evidence is not completely consistent, however, as some studies suggest that those with weak intentions may benefit more from planning interventions (Browne \& Chan, 2012; Rutter et al., 2006; Sheeran \& Orbell, 2000). But it is important to note that there is no research suggesting that planning interventions will be effective in the absence of an intention. It seems that the conditions under which low intenders, and those with weak intentions, are likely to respond to planning interventions have yet to be elucidated and it is likely that moderator variables exist that determine when implementation intentions are likely to be effective for this group.

Importantly, there is a relative dearth of research examining the effectiveness of planning interventions among groups with very low intentions, low motivation, and high resistance to change their behaviour. The likelihood is that implementation intention and other planning techniques would have little resonance, or make little sense, to these individuals who are not likely to have given consideration to changing their behaviour. This has not, however, been tested formally. Such a test would be an important endeavour as these kinds of intervention may be rolled out as part of large scale public health campaigns, and although they may cause behaviour change at the population level, it would be necessary to evaluate whether the changes were confined to certain groups, such as those who have formed intentions but perhaps did not have the resources or capacity to act on their intentions (Orbell \& Sheeran, 1998). Furthermore, such research may also shed light on the mechanisms underpinning 
planning interventions. Given research demonstrating that even responding to 'minimal' components relating to an intervention, such as responding to questionnaire items (Godin, Belanger-Gravel, Amireault, Vohl, \& Perusse, 2010; Godin et al., 2008), may enhance behavioural engagement, it may not be unreasonable to expect that the completion of planning exercises would have some effect and may be sufficient to evoke a small, albeit significant, change in behaviour. Thus far answers to these questions have not been forthcoming and we look to future investigations to elucidate the role that planning interventions may play among 'low intenders' or 'unmotivated' individuals, as well as among who are resistant to change.

\section{Intervention Design Issues}

\section{Sustainability of Behaviour Change}

While there are occasions where it is desirable for interventions to lead to a one-off behaviour change such as getting vaccinated or donating blood, it is frequently desirable for health interventions to lead to sustained behaviour change over time and to produce outcomes of clinical and practical significance (Hagger \& Chatzisarantis, 2009). It is therefore important that planning interventions compare favourably with this aim. The vast majority of interventions adopting implementation intentions and action planning have only evaluated their effectiveness over relatively short follow-up periods with few studies investigating the sustainability of effects over periods of one-year or greater (Prestwich et al., 2012). In fact, we identified only four controlled trials evaluating long-term effects of implementation intentions or action planning interventions and targeting health behaviour change or health-related outcomes $^{6}$. Sustainable effects of the interventions were found on behavioural outcomes in three of the interventions targeting smoking cessation (Conner \& Higgins, 2010), blood donation (Godin, Sheeran, et al., 2010), and oral contraceptive use (Martin et al., 2009). A fourth focusing on dietary fat intake found no significant effects on behaviour change at longterm follow-up (Scholz, Ochsner, \& Luszczynska, in press). Importantly, the three trials

\footnotetext{
${ }^{6}$ According to a Web of Science (Thomson ISI, 2012) literature search for published items between 1997 and September 2012. The search aimed at studies evaluating long-term effects of implementation intentions or action planning interventions of at least 12 months post-intervention. We only considered studies evaluating the effects of planning as a stand-alone behaviour-change technique (i.e., not combined with other techniques) so that the effects could be attributed directly to the planning intervention.
} 
showing sustainable effects collected objective and self-reported measures of behaviour, an indication of quality given the over-reliance on self-report behavioural measures in research on planning interventions.

A striking similarity among these studies is that all incorporated additional components alongside the standard planning intervention. Conner and Higgins (2010) found durable effects of a planning intervention (consisting of seven sessions delivered over 24 months) on smoking among adolescents. The planning intervention included five prespecified examples of refusal responses, an option to choose and write down an individual response, and a requirement to sign the planning form (Conner \& Higgins, 2010). The intervention group exhibited lower frequency of smoking and expired carbon monoxide at 24 months post-intervention. Godin et al. (2010) targeted first time blood donors and asked them to read and memorise two prespecified 'if-then' plans that included an environmental cue (e.g., a phone call from a specific organisation) and self-generated reactions. Compared to controls, participants allocated to the intervention group were more likely to register for blood donation at 1-year follow-up (Godin, Sheeran, et al., 2010). Martin et al. (2009) approached teenage women visiting a family planning clinic and invited them to write down when, where, and how they would take a contraceptive pill and how they would overcome a series of 12 pre-specified barriers to taking the pill. Intervention participants had higher rates of consulting for contraceptive pills compared to control group participants but lower rates of consulting for emergency contraception and positive pregnancy testing. Finally, Scholz et al. (in press) required a sample of obese participants to form action and coping plans, with participants receiving one face-toface session and weekly planning sheets for up to nine weeks. No significant intervention effects were found on self-reported dietary fat intake.

The inclusion of additional components alongside the planning interventions limits the extent to which generalisable conclusions can be drawn from the results, but indicates a recognition of the need for planning components to be augmented or complemented in some way in order to bring about sustainable change. One possible reason for this may be that researchers acknowledge that long-term interventions are costly in terms of finance, human resources, and time and may baulk at relying exclusively on the 'minimal' strategies proposed 
by the planning interventions and feel the need to 'beef up' their interventions by other means to ensure that they get sustainable effects. One approach would be to adopt a factorial, multiarm randomised controlled design which would allow comparison of the sustainability of the effects of the planning techniques alongside planning interventions augmented with other components. This would directly address recent calls to disentangle the active components of the interventions that result in sustainable effects on long-term health outcomes (Michie, 2008). Further, such factorial designs provide essential empirical evidence for developing complex, multi-component health promotion programs.

\section{Intervention Fidelity}

In order to fully establish the effectiveness of a health-behaviour intervention, research needs to demonstrate the extent to which individuals comply with the intervention and the precision which with the intervention has been implemented by those responsible for its delivery. This is frequently referred to as the fidelity of the intervention and is a priority for intervention designers seeking to maximise the effectiveness and reach of their interventions in public health settings. With respect to research on interventions adopting planning as a behaviour-change strategy, it is important that sufficient checks are put in place in the intervention protocol in order to ensure that it has been implemented effectively. Most of the planning interventions are delivered in print form and require participants to read text, respond to questions, and, in most cases, engage in planning exercises. Means to evaluate whether participants have engaged with these exercises are relatively straightforward, often requiring participants to self-report the extent of their planning. However, such items alone do not necessarily confirm compliance with the exercises. A more potent means is to conduct a content analysis of responses to the planning exercises following the precedent set by Orbell et al. (1997), an exercise that is seldom conducted in planning studies (e.g., Armitage, 2004; Jackson et al., 2005; Koestner, Lekes, Powers, \& Chicoine, 2002; Prestwich et al., 2003). Identifying whether participants have formed clear plans in accordance with protocol (e.g., writing down an 'if-then' plan making reference to a salient cue and the target behaviour) will provide confirmation of compliance and an absence of a plan or an inappropriate response may raise questions as to the extent to which individuals have complied. Furthermore, if compliance 
is low, then this may bring in to question to fidelity of the intervention and highlight the need for modifications such as better instructions or an alternative means of delivery. It may therefore be important to pilot protocols of planning interventions with these checks in mind in order to ensure that the fidelity of the intervention is satisfactory before rolling it out to a larger sample or population. This will ensure that a costly revision of a low-fidelity intervention is avoided and the evaluation of the effectiveness of the intervention is unconfounded by compliance and fidelity issues. Researchers are therefore encouraged to ensure that sufficient fidelity checks are including in planning intervention protocols to ensure that the effectiveness and mechanisms are unconfounded by low compliance.

\section{The Way Forward: Summary and Recommendations for Future Research}

The purpose of this article was to present an evidence-based position statement on the effectiveness of interventions adopting two key planning techniques, implementation intentions and action planning, to promote behaviour in health contexts. We aimed to identify the key similarities and differences in the two approaches, identify the gaps in the literature and areas in need of further research inquiry, and highlight trends that indicate good practice in planning interventions. We identified four key areas relevant to the effectiveness of planning interventions and the current state of the literature: (1) issues with definition and conceptualisation; (2) format and measurement; (3) mechanisms and processes; and (4) design issues. Our analysis of each area highlighted the features of planning interventions that may be most effective in changing behaviour based on what is currently known and informed the identification of the key outstanding research questions that require resolution in order to gain a better understanding of the way planning interventions work and how their effectiveness in producing health behaviour change can be maximised.

In terms of definitions, implementation intentions and action planning share common attributes in that they specify self-relevant cues-to-action identifying 'when' and 'where' the behaviour will be enacted, usually in an 'if-then' format. Implementation intentions comprise exclusively of 'if-then' plans, while the definition of action planning is less concrete. The technique differs from implementation intentions in that it tends to include additional planning 
components to cope with barriers or contingencies or to assist with the initiation of the behaviour.

From the perspective of format and content, we provide the following recommendations to researchers developing interventions containing planning components based on the evidence discussed in the current article. Planning interventions should:

- Adopt 'if-then' rather than 'global' formatted plans (e.g., Chapman et al., 2009)

- Contain personally-relevant, accessible cues linked to behavioural outcome, based on pilot data to identify appropriate cues (e.g., Adriaanse et al., 2009)

- Include examples of cues likely to be salient based on pilot data (e.g., Luszczynska et al., 2007)

- Be researcher-guided rather than user-guided (e.g., Armitage, 2009)

- Adopt a dyadic or collaborative approach (e.g., Prestwich et al., 2005)

- Include 'booster' reminders (e.g., Schwerdtfeger et al., 2012)

- Include additional multiple planning components to cope with contingencies (e.g., coping plans to deal with barriers) (e.g., De Vet et al., 2009)

- Include means to promote motivation and self-efficacy alongside planning components, particularly in populations resistant to change, with strong habits and weak intentions (e.g., Milne et al., 2002)

The above set of recommendations very much represents a 'wish-list' of planning intervention components based on the most recent available evidence demonstrating their effectiveness. Of course, from a pragmatic perspective, it may not be convenient or economically viable to account for all of these recommendations in a single intervention. Furthermore, many of these recommendations are based on evidence from a relatively small subset of studies and may be subject to modification or revision as new evidence comes to light. Related to this, it is clear from the literature reviewed in the current paper and recent systematic reviews and meta-analyses of research on planning interventions that better quality data is required (Adriaanse, Vinkers, et al., 2011; Bélanger-Gravel et al., 2013). This includes the adoption of objective measures of behavioural outcomes, making protocols available and easily accessible (e.g., in e form of e-supplements) for scientific purposes, and the formal 
registration of trial protocols to maximise transparency and accountability. There is also a need for future systematic reviews and meta-analyses to pay greater attention to these quality criteria that may potentially resolve the observed heterogeneity in the effect sizes of planning interventions in health contexts. We do acknowledge, however, that applying stringent quality criteria to previous systematic reviews would mean many studies in the current literature would be excluded.

In addition to evidence-based guidelines for the content of planning interventions, the current paper highlights the evidence base for recommending the design features of studies that test the effectiveness of planning interventions. Based on current research findings, intervention studies adopting implementation intention and action planning components need to include the following design elements to sufficiently evaluate their effectiveness:

- A randomised controlled trial design (e.g., Conner \& Higgins, 2010) and inclusion of study protocol on a public trials registry prior to data collection (De Vet et al., 2009)

- Measures of mediators to explain mechanisms (e.g. planning measures; (e.g., Luszczynska et al., 2007)

- Appropriate objective behavioural measures from social-cognitive models matched with behaviour specified in plan (e.g., Armitage, 2004)

- Clear and comprehensive reporting of intervention protocols (e.g., Armitage \& Arden, 2010)

- Appropriate fidelity measures (e.g., Hagger, Lonsdale, \& Chatzisarantis, 2012)

- Relevant comparison/control group(s) (e.g., Godin et al., 2008)

- Long-term follow-up measures ( $\geq 12$ months post-intervention) of relevant outcome variables, particularly related to the target action or behaviour, to ensure sustainability of intervention (e.g., Martin et al., 2009)

Again, researchers need to make executive decisions as to which components should be included in their intervention trials. The above very much represents a 'gold standard' when it comes to evaluating the effectiveness of interventions, but constraints on time and funding may necessitate the omission of some components. For example, although they are needed, we 
recognise that not all planning intervention trials will be able to incorporate long-term followup of outcome measures.

In addition to the development of 'gold standard' trials to evaluate the effectiveness and mechanisms of planning in health contexts, we have discussed a number of 'gaps' in the literature on planning interventions. Priority areas of research are should be the identification of the mechanisms that underpin the effects of planning interventions on behavioural outcomes and the contexts and conditions that are likely to magnify or diminish the effects of such interventions on behaviour - in short, the mediating and moderating factors, respectively, of the effects of the planning interventions. In terms of moderators, future research should aim to examine the moderating effects of (a) individual difference variables related to information processing and behavioural regulation such as impulsivity and executive functioning (e.g., Churchill \& Jessop, 2010; Hall et al., 2012); (b) habit strength (e.g., Adriaanse, Gollwitzer, et al., 2011); (c) intention strength and 'low intenders' (Sheeran, Webb, et al., 2005); and (d) different modes of delivery (e.g., written text vs. online vs. test message; Craciun et al., 2012; Luszczynska et al., 2007; Prestwich et al., 2009) on the impact of planning interventions on behavioural outcomes. In terms of mediation analyses, researchers should make it standard practice to include a mediation analysis using measures of extent of planning to confirm its role in explaining intervention effects. It would also be useful to incorporate additional components to bolster implementation intentions such as coping planning in studies with factorial designs to establish the potential multiple processes operating in terms of changing behaviour (Scholz et al., in press). Given that implementation intentions are likely to have different mediators relative to techniques like coping planning, one would expect that the effects of each intervention would be mediated by different constructs. Research is needed to test the hypothesis that the different planning components affect behavioural outcomes via different mechanisms (Luszczynska, 2006).

Our intention in the current analysis was to provide a comprehensive overview of the research of planning interventions in health behaviour, outline the limitations and gaps in current knowledge, and provide a basis for future research inquiry. We have provided a set of recommendations for the content and design of planning interventions that represent best 
practice according to the currently available evidence. Further, we have identified a number of outstanding questions that need to be addressed in future research. We also acknowledge the heterogeneity in effect sizes and variable quality of the research in current literature and call for high-quality randomised trials using planning techniques in multiple behavioural contexts. This will contribute to the growing body of evidence suggesting that planning interventions are simple and effective techniques for changing health behaviour. Given the pivotal role of replication in psychology and the necessity to provide converging evidence that support the generalisability of such effects (Pashler \& Harris, 2012; Ritchie, Wiseman, \& French, 2012), such research is essential. Parallel to high-quality replication trials, blue skies research examining the mechanisms and processes for which there is little or no evidence, will advance knowledge and understanding of the way in which planning interventions work and elucidate the conditions, content, and design of interventions that will maximise their effectiveness. 


\section{References}

Abraham, C., Kok, G., Schaalma, H. P., \& Luszczynska, A. (2011). Health promotion. In P. R. Martin, F. M. Cheung, M. C. Knowles, M. Kyrios, L. Littlefield, J. B. Overmier \& J. M. Prieto (Eds.), Handbook of applied psychology (pp. 83-111). Chichester, UK: Wiley-Blackwell.

Abraham, C., Southby, L., Quandte, S., Krahe, B., \& van der Sluijs, W. (2007). What's in a leaflet? Identifying research-based persuasive messages in European alcohol-education leaflets. Psychology \& Health, 22, 31-60. doi: 10.1080/14768320600774405

Adriaanse, M. A., de Ridder, D. T. D., \& de Wit, J. B. F. (2009). Finding the critical cue: Implementation intentions to change one's diet work best when tailored to personally relevant reasons for unhealthy eating. Personality and Social Psychology Bulletin, 35, 60-71. doi: 10.1177/0146167208325612

Adriaanse, M. A., Gollwitzer, P. M., De Ridder, D. T. D., de Wit, J. B. F., \& Kroese, F. M. (2011). Breaking habits with implementation intentions: A test of underlying processes. Personality and Social Psychology Bulletin, 37, 502-513. doi: 10.1177/0146167211399102

Adriaanse, M. A., Oettingen, G., Gollwitzer, P. M., Hennes, E. P., de Ridder, D. T. D., \& de Wit, J. B. F. (2010). When planning is not enough: Fighting unhealthy snacking habits by mental contrasting with implementation intentions (MCII). European Journal of Social Psychology, 40, 1277-1293. doi: 10.1002/ejsp.730

Adriaanse, M. A., van Oosten, J. M. F., de Ridder, D. T. D., de Wit, J. B. F., \& Evers, C. (2011). Planning what not to eat: Ironic effects of implementation intentions negating unhealthy habits. Personality and Social Psychology Bulletin, 37, 69-81. doi: 10.1177/0146167210390523

Adriaanse, M. A., Vinkers, C. D. W., De Ridder, D. T. D., Hox, J. J., \& De Wit, J. B. F. (2011). Do implementation intentions help to eat a healthy diet? A systematic review and meta-analysis of the empirical evidence. Appetite, 56, 183-193. doi: 10.1016/j.appet.2010.10.012

Ajzen, I. (1985). From intentions to actions: A theory of planned behavior. In J. Kuhl \& J. Beckmann (Eds.), Action-control: From cognition to behavior (pp. 11-39). Heidelberg: Springer.

Andersson, E. K., \& Moss, T. P. (2011). Imagery and implementation intention: A randomised controlled trial of interventions to increase exercise behaviour in the general population. Psychology of Sport and Exercise, 12, 63-70. doi: 10.1016/j.psychsport.2010.07.004

Arbour, K. P., \& Martin Ginis, K. A. (2009). A randomised controlled trial of the effects of implementation intentions on women's walking behaviour. Psychology \& Health, 24, 49-65. doi: 10.1080/08870440801930312

Arden, M. A., \& Armitage, C. J. (2012). A volitional help sheet to reduce binge drinking in students: A randomized exploratory trial. Alcohol and Alcoholism, 47, 156-159. doi: 10.1093/alcalc/agr164

Armitage, C. J. (2004). Evidence that implementation intentions reduce dietary fat intake: A randomized trial. Health Psychology, 23, 319-323.

Armitage, C. J. (2007). Effects of an implementation intention-based intervention on fruit consumption. Psychology \& Health, 22, 917-928. doi: 10.1080/14768320601070662

Armitage, C. J. (2008). A volitional help sheet to encourage smoking cessation: A randomized exploratory trial. Health Psychology, 27, 557-566. doi: 10.1037/0278-6133.27.5.557

Armitage, C. J. (2009). Effectiveness of experimenter-provided and self-generated implementation intentions to reduce alcohol consumption in a sample of the general population: A randomized exploratory trial. Health Psychology, 28, 545-553. doi: 10.1037/a0015984 
Armitage, C. J., \& Arden, M. A. (2008). How useful are the stages of change for targeting interventions? Randomized test of a brief intervention to reduce smoking. Health Psychology, 27, 789-798. doi: 10.1037/0278-6133.27.6.789

Armitage, C. J., \& Arden, M. A. (2010). A volitional help sheet to increase physical activity in people with low socioeconomic status: A randomised exploratory trial. Psychology \& Health, 25, 1129 - 1145. doi: 10.1080/08870440903121638

Armitage, C. J., \& Conner, M. (2000). Social cognition models and health behaviour: A structured review. Psychology and Health, 15, 173-189.

Armitage, C. J., \& Conner, M. (2001). Efficacy of the theory of planned behaviour: A metaanalytic review. British Journal of Social Psychology, 40, 471-499.

Bagozzi, R. P., Dholakia, U. M., \& Basuroy, S. (2003). How effortful decisions get enacted: The motivating role of decision processes, desires, and anticipated emotions. Journal of Behavioral Decision Making, 16, 273-295. doi: 10.1002/bdm.446

Bagozzi, R. P., \& Edwards, E. A. (1998). Goal setting and goal pursuit in the regulation of body weight. Psychology and Health, 13, 593-621.

Barg, C. J., Latimer, A. E., Pomery, E. A., Rivers, S. E., Rench, T. A., Prapavessis, H., \& Salovey, P. (2012). Examining predictors of physical activity among inactive middleaged women: An application of the health action process approach. Psychology \& Health, 27, 829-845. doi: 10.1080/08870446.2011.609595

Bartholomew, L. K., Parcel, G. S., Kok, G., Gottlieb, N. H., \& Fernández, M. E. (2011). Planning health promotion programs: An Intervention Mapping approach (3rd ed.). San Francisco, CA: Jossey-Bass.

Bayer, U. C., \& Gollwitzer, P. M. (2007). Boosting scholastic test scores by willpower: The role of implementation intentions. Self and Identity, 6, 1-19. doi: $10.1080 / 15298860600662056$

Bélanger-Gravel, A., Godin, G., \& Amireault, S. (2013). A meta-analytic review of the effect of implementation intentions on physical activity. Health Psychology Review, 7, 23-54. doi: 10.1080/17437199.2011.560095

Brandstätter, V., Lengfelder, A., \& Gollwitzer, P. M. (2001). Implementation intentions and efficient action initiation. Journal of Personality and Social Psychology, 81, 946-960. doi: 10.1037//0022-3514.81.5.946

Browne, J. L., \& Chan, A. Y. C. (2012). Using the Theory of Planned Behaviour and implementation intentions to predict and facilitate upward family communication about mammography. Psychology \& Health, 27, 655-673. doi: 10.1080/08870446.2011.615396

Burkert, S., Knoll, N., Luszczynska, A., \& Gralla, O. (2012). The interplay of dyadic and individual planning of pelvic-floor exercise in prostate-cancer patients following radical prostatectomy. Journal of Behavioral Medicine, 35, 305-317. doi: 10.1007/s10865-0129416-2

Burkert, S., Scholz, U., Gralla, O., \& Knoll, N. (2011). Dyadic planning of health-behavior change after prostatectomy: A randomized-controlled planning intervention. Social Science \& Medicine, 73, 783-792. doi: 10.1016/j.socscimed.2011.06.016

Carraro, N., \& Gaudreau, P. (2013). Spontaneous and experimentally induced action planning and coping planning for physical activity: A meta-analysis. Psychology of Sport and Exercise, 14, 228-248. doi: 10.1016/j.psychsport.2012.10.004

Chapman, J., \& Armitage, C. J. (2010). Evidence that boosters augment the long-term impact of implementation intentions on fruit and vegetable intake. Psychology \& Health, 25, 365-381. doi: 10.1080/08870440802642148

Chapman, J., Armitage, C. J., \& Norman, P. (2009). Comparing implementation intention interventions in relation to young adults' intake of fruit and vegetables. Psychology and Health, 24, 317-332. doi: 10.1080/08870440701864538 
Chatzisarantis, N. L. D., Hagger, M. S., \& Wang, C. K. J. (2010). Evaluating the effects of implementation intention and self-concordance on behaviour. British Journal of Psychology, 101, 705-718. doi: 10.1348/000712609X481796

Churchill, S., \& Jessop, D. (2010). Spontaneous implementation intentions and impulsivity: Can impulsivity moderate the effectiveness of planning strategies? British Journal of Health Psychology, 15, 529-541. doi: 10.1348/135910709x475423

Churchill, S., \& Jessop, D. C. (2011). Too impulsive for implementation intentions? Evidence that impulsivity moderates the effectiveness of an implementation intention intervention. Psychology \& Health, 26, 517-530. doi: 10.1080/08870441003611536

Conner, M., \& Higgins, A. R. (2010). Long-term effects of implementation intentions on prevention of smoking uptake among adolescents: A cluster randomized controlled trial. Health Psychology, 29, 529-538. doi: 10.1037/a0020317

Conner, M., Sandberg, T., \& Norman, P. (2010). Using action planning to promote exercise behavior. Annals of Behavioral Medicine, 40, 65-76. doi: 10.1007/s12160-010-9190-8

Craciun, C., Schuz, N., Lippke, S., \& Schwarzer, R. (2012). Facilitating sunscreen use in women by a theory-based online intervention: A randomized controlled trial. Journal of Health Psychology, 17, 207-216. doi: 10.1177/1359105311414955

de Vet, E., Gebhardt, W. A., Sinnige, J., Van Puffelen, A., Van Lettow, B., \& de Wit, J. B. F. (2011). Implementation intentions for buying, carrying, discussing and using condoms: The role of the quality of plans. Health Education Research, 26, 443-455. doi: 10.1093/her/cyr006

De Vet, E., Oenema, A., Sheeran, P., \& Brug, J. (2009). Should implementation intentions interventions be implemented in obesity prevention: The impact of if-then plans on daily physical activity in Dutch adults. International Journal of Behavioral Nutrition and Physical Activity, 6, 11. doi: 10.1186/1479-5868-6-11

de Vries, H., Mesters, I., van de Steeg, H., \& Honing, C. (2005). The general public's information needs and perceptions regarding hereditary cancer: An application of the Integrated Change Model. Patient Education and Counseling, 56, 154-165. doi: 10.1016/j.pec.2004.01.002

de Vries, H., Mudde, A., Leijs, I., Charlton, A., Vartiainen, E., Buijs, G., . . Kremers, S. (2003). The European Smoking Prevention Framework Approach (EFSA): An example of integral prevention. Health Education Research, 18, 611-626.

Dekker, J. (2008). Theories in behavioral medicine. International Journal of Behavioral Medicine, 15, 1-3.

Dohnke, B., Nowossadeck, E., \& Muller-Fahrnow, W. (2010). Motivation and Participation in a Phase III Cardiac Rehabilitation Programme: An Application of the Health Action Process Approach. Research in Sports Medicine, 18, 219-235. doi: 10.1080/15438627.2010.510032

Doll, J., \& Ajzen, I. (1992). Accessibility and stability of predictors in the Theory of Planned Behavior. Journal of Personality and Social Psychology, 63, 754-765.

Fishbein, M., \& Ajzen, I. (2009). Predicting and changing behavior: The reasoned action approach. New York: Psychology Press.

Fleig, L., Pomp, S., Parschau, L., Barz, M., Lange, D., Schwarzer, R., \& Lippke, S. (2013). From intentions via planning and behavior to physical exercise habits. Psychology of Sport and Exercise, 14, 632-639. doi: 10.1016/j.psychsport.2013.03.006

Fuchs, R., Goehner, W., \& Seelig, H. (2011). Long-term effects of a psychological group intervention on physical exercise and health: The MoVo concept. Journal of Physical Activity \& Health, 8, 794-803.

Gallo, I. S., McCulloch, K. C., \& Gollwitzer, P. M. (2012). Differential effects of various types of implementation intentions on the regulation of disgust. Social Cognition, 30, 1-17. 
Gawrilow, C., Gollwitzer, P. M., \& Oettingen, G. (2011a). If-then plans benefit delay of gratification performance in children with and without ADHD. Cognitive Therapy and Research, 35, 442-455. doi: 10.1007/s10608-010-9309-z

Gawrilow, C., Gollwitzer, P. M., \& Oettingen, G. (2011b). If-then plans benefit executive functions in children with ADHD. Journal of Social and Clinical Psychology, 30, 616646.

Gellert, P., Ziegelmann, J. P., Lippke, S., \& Schwarzer, R. (2012). Future time perspective and health behaviors: Temporal framing of self-regulatory processes in physical exercise and dietary behaviors. Annals of Behavioral Medicine, 43, 208-218. doi: 10.1007/s12160-011-9312-y

Godin, G., Belanger-Gravel, A., Amireault, S., Gallani, M., Vohl, M. C., \& Perusse, L. (2010). Effect of implementation intentions to change behaviour: Moderation by intention stability. Psychological Reports, 106, 147-159. doi: 10.2466/pr0.106.1.147-159

Godin, G., Belanger-Gravel, A., Amireault, S., Vohl, M.-C., \& Perusse, L. (2010). The effect of mere-measurement of cognitions on physical activity behavior: A randomized controlled trial among overweight and obese individuals. International Journal of Behavioral Nutrition and Physical Activity, 8, 2. doi: 10.1186/1479-5868-8-2

Godin, G., Conner, M., \& Sheeran, P. (2005). Bridging the intention-behaviour 'gap': The role of moral norm. British Journal of Social Psychology, 44, 497-512.

Godin, G., Sheeran, P., Conner, M., Delage, G., Germain, M., Bélanger-Gravel, A., \& Naccache, H. (2010). Which survey questions change behavior? Randomized controlled trial of mere measurement interventions. Health Psychology, 29, 636-644. doi: $10.1037 / \mathrm{a} 0021131$

Godin, G., Sheeran, P., Conner, M., \& Germain, M. (2008). Asking questions changes behavior: Mere measurement effects on frequency of blood donation. Health Psychology, 27, 179-184. doi: 10.1037/0278-6133.27.2.179

Gollwitzer, P. M. (1999). Implementation intentions: Strong effects of simple plans. American Psychologist, 54, 493-503.

Gollwitzer, P. M., \& Sheeran, P. (2006). Implementation intentions and goal achievement: A meta-analysis of effects and processes. Advances in Experimental Social Psychology, 38, 69-119. doi: 10.1013/S0065-2601(06)38002-1

Green, L. W., \& Kreuter, M. W. (2004). Health promotion and planning: An educational and ecological approach (4th ed.). New York, NY: McGraw-Hill.

Guillaumie, L., Godin, G., Manderscheid, J. C., Spitz, E., \& Muller, L. (2012). The impact of self-efficacy and implementation intentions-based interventions on fruit and vegetable intake among adults. Psychology \& Health, 27, 30-50. doi:

$10.1080 / 08870446.2010 .541910$

Hagger, M. S. (2009). Theoretical integration in health psychology: Unifying ideas and complimentary explanations. British Journal of Health Psychology, 14, 189-194. doi: 10.1348/135910708X397034

Hagger, M. S. (2010a). Current issues and new directions in psychology and health: Physical activity research showcasing theory into practice. Psychology and Health, 25, 1-5. doi: 10.1080/08870440903268637

Hagger, M. S. (2010b). Self-regulation: An important construct in health psychology research and practice. Health Psychology Review, 4, 57-65. doi: 10.1080/17437199.2010.503594

Hagger, M. S., Anderson, M., Kyriakaki, M., \& Darkings, S. (2007). Aspects of identity and their influence on intentional behaviour: Comparing effects for three health behaviours. Personality and Individual Differences, 42, 355-367. doi: 10.1016/j.paid.2006.07.017

Hagger, M. S., \& Chatzisarantis, N. L. D. (2009). Assumptions in research in sport and exercise psychology. Psychology of Sport and Exercise, 10, 511-519. doi: 10.1016/j.psychsport.2009.01.004 
Hagger, M. S., \& Chatzisarantis, N. L. D. (2013). An integrated theoretical model of physical activity behavior change. Perth, Australia: Curtin University.

Hagger, M. S., Chatzisarantis, N. L. D., \& Biddle, S. J. H. (2002). A meta-analytic review of the theories of reasoned action and planned behavior in physical activity: Predictive validity and the contribution of additional variables. Journal of Sport and Exercise Psychology, 24, 3-32.

Hagger, M. S., Keatley, D. A., \& Chan, D. K. C. (in press). CALO-RE Taxonomy of Behavior Change Techniques. In R. C. Eklund \& G. T. Tenenbaum (Eds.), Encyclopedia of Sport and Exercise Psychology. Thousand Oaks, CA: Sage.

Hagger, M. S., Lonsdale, A., Koka, A., Hein, V., Pasi, H., Lintunen, T., \& Chatzisarantis, N. L. D. (2012). An intervention to reduce alcohol consumption in undergraduate students using implementation intentions and mental simulations: A cross-national study. International Journal of Behavioral Medicine, 19, 82-96. doi: 10.1007/s12529-0119163-8

Hagger, M. S., Lonsdale, A. J., \& Chatzisarantis, N. L. D. (2012). A theory-based intervention to reduce alcohol drinking in excess of guideline limits among undergraduate students. British Journal of Health Psychology, 17, 18-43. doi: 10.1111/j.20448287.2010.02011.x

Hagger, M. S., Wood, C., Stiff, C., \& Chatzisarantis, N. L. D. (2009). The strength model of self-regulation failure and health-related behavior. Health Psychology Review, 3, 208238. doi: 10.1080/17437190903414387

Hagger, M. S., Wood, C., Stiff, C., \& Chatzisarantis, N. L. D. (2010). Ego depletion and the strength model of self-control: A meta-analysis. Psychological Bulletin, 136, 495-525. doi: $10.1037 / \mathrm{a} 0019486$

Hall, P. A., \& Fong, G. T. (2010). Temporal self-regulation theory: Looking forward. Health Psychology Review, 4, 83-92. doi: 10.1080/17437199.2010.487180

Hall, P. A., Fong, G. T., Epp, L. J., \& Elias, L. J. (2008). Executive function moderates the intention-behavior link for physical activity and dietary behavior. Psychology \& Health, 23, 309-326. doi: 10.1080/14768320701212099

Hall, P. A., Zehr, C. E., Ng, M., \& Zanna, M. P. (2012). Implementation intentions for physical activity in supportive and unsupportive environmental conditions: An experimental examination of intention-behavior consistency. Journal of Experimental Social Psychology, 48, 432-436. doi: 10.1016/j.jesp.2011.09.004

Heckhausen, H., \& Gollwitzer, P. M. (1987). Thought contents and cognitive functioning in motivational and volitional states of mind. Motivation and Emotion, 11, 101-120. doi: 10.1007/BF00992338

Hofmann, W., Friese, M., \& Wiers, R. W. (2011). Impulsive processes in the self-regulation of health behaviour: Theoretical and methodological considerations in response to commentaries. Health Psychology Review, 5, 162-171. doi: 10.1080/17437199.2011.565593

Jackson, C., Lawton, R., Knapp, P., Raynor, D. K., Conner, M., Lowe, C., \& Closs, S. J. (2005). Beyond intention: Do specific plans increase health behaviours in patients in primary care? A study of fruit and vegetable consumption. Social Science \& Medicine, 60, 2383-2391.

Keatley, D. A., Clarke, D. D., \& Hagger, M. S. (2012). Investigating the predictive validity of implicit and explicit measures of motivation on condom use, physical activity, and healthy eating. Psychology \& Health, 27, 550-569. doi: 10.1080/08870446.2011.605451

Keatley, D. A., Clarke, D. D., \& Hagger, M. S. (2013). The predictive validity of implicit measures of self-determined motivation across health-related behaviours. British Journal of Health Psychology, 18, 2-17. doi: 10.1111/j.2044-8287.2011.02063.x 
Knauper, B., Pillay, R., Lacaille, J., McCollam, A., \& Kelso, E. (2011). Replacing craving imagery with alternative pleasant imagery reduces craving intensity. Appetite, 57, 173178. doi: 10.1016/j.appet.2011.04.021

Knauper, B., Roseman, M., Johnson, P. J., \& Krantz, L. H. (2009). Using mental imagery to enhance the effectiveness of implementation intentions. Current Psychology, 28, 181186. doi: $10.1007 / \mathrm{s} 12144-009-9055-0$

Koestner, R., Horberg, E. J., Gaudreau, P., Powers, T., Di Dio, P., Bryan, C., . . Salter, N. (2006). Bolstering implementation plans for the long haul: The benefits of simultaneously boosting self-concordance or self-efficacy. Personality and Social Psychology Bulletin, 32, 1547-1558.

Koestner, R., Lekes, N., Powers, T. A., \& Chicoine, E. (2002). Attaining personal goals: Selfconcordance plus implementation intentions equals success. Journal of Personality and Social Psychology, 83, 231-244.

Koring, M., Parschau, L., Lange, D., Fleig, L., Knoll, N., \& Schwarzer, R. (2013). Preparing for physical activity: Pedometer acquisition as a self-regulatory strategy. Applied Psychology: Health and Well-Being, 5, 136-147. doi: 10.1111/aphw.12003

Leventhal, H., Singer, R., \& Jones, S. (1965). Affects of fear and specificity of recommendation upon attitudes and behavior. Journal of Personality and Social Psychology, 34, 20-29.

Lewin, K. (1951). Intention, will, and need. In D. Rapaport (Ed.), Organisation and pathology of thought (pp. 95-153). New York: Columbia University Press.

Luszczynska, A. (2006). An implementation intentions intervention, the use of a planning strategy, and physical activity after myocardial infarction. Social Science \& Medicine, 62, 900-908. doi: 10.1016/j.socscimed.2005.06.043

Luszczynska, A., Cao, D. S., Mallach, N., Pietron, K., Mazurkiewicz, M., \& Schwarzer, R. (2010). Intentions, planning, and self-efficacy predict physical activity in Chinese and Polish adolescents: Two moderated mediation analyses. International Journal of Clinical and Health Psychology, 10, 265-278.

Luszczynska, A., \& Schwarzer, R. (2003). Planning and self-efficacy in the adoption and maintenance of breast self-examination: A longitudinal study on self-regulatory cognitions. Psychology and Health, 18, 93-108.

Luszczynska, A., Schwarzer, R., Lippke, S., \& Mazurkiewicz, M. (2011). Self-efficacy as a moderator of the planning-behaviour relationship in interventions designed to promote physical activity. Psychology \& Health, 26, 151-166. doi: 10.1080/08870446.2011.531571

Luszczynska, A., Sobczyk, A., \& Abraham, C. (2007). Planning to lose weight: Randomized controlled trial of an implementation intention prompt to enhance weight reduction among overweight and obese women. Health Psychology, 26, 507-512. doi: 10.1037/0278-6133.26.4.507

Martin, J., Sheeran, P., Slade, P., Wright, A., \& Dibble, T. (2009). Implementation intention formation reduces consultations for emergency contraception and pregnancy testing among teenage women. Health Psychology, 28, 762-769. doi: 10.1037/a0016200

McEachan, R. R. C., Conner, M. T., Taylor, N., \& Lawton, R. J. (2012). Prospective prediction of health-related behaviors with the Theory of Planned Behavior: A meta-analysis. Health Psychology Review, 5, 97-144. doi: 10.1080/17437199.2010.521684

Michie, S. (2008). What works and how? Designing more effective interventions needs answers to both questions. Addiction, 103, 886-887. doi: 10.1111/j.13600443.2007.02112.x

Michie, S., \& Abraham, C. (2008). Advancing the science of behaviour change: A plea for scientific reporting. Addiction, 103, 1409-1410. doi: 10.1111/j.1360-0443.2008.02291.x 
Milkman, K. L., Beshears, J., Choi, J. J., Laibson, D., \& Madrian, B. C. (2011). Using implementation intentions prompts to enhance influenza vaccination rates. Proceedings of the National Academy of Sciences of the United States of America, 108, 1041510420. doi: 10.1073/pnas.1103170108

Milne, S. E., Orbell, S., \& Sheeran, P. (2002). Combining motivational and volitional interventions to promote exercise participation: Protection motivation theory and implementation intentions. British Journal of Health Psychology, 7, 163-184.

Mullan, B., Wong, C., Allom, V., \& Pack, S. L. (2011). The role of executive function in bridging the intention-behaviour gap for binge-drinking in university students. Addictive Behaviors, 36, 1023-1026. doi: 10.1016/j.addbeh.2011.05.012

O'Sullivan, I., Orbell, S., Rakow, T., \& Parker, R. (2004). Prospective research in health service settings: Health psychology, science and the 'Hawthorne' effect. Journal of Health Psychology, 9, 355-359. doi: 10.1177/1359105304042345

Ochsner, S., Scholz, U., \& Hornung, R. (2013). Testing phase-specific self-efficacy beliefs in the context of dietary behaviour change. Applied Psychology: Health and Well-Being, 5, 99-117. doi: 10.1111/j.1758-0854.2012.01079.x

Orbell, S., Hodgkins, S., \& Sheeran, P. (1997). Implementation intentions and the Theory of Planned Behavior. Personality and Social Psychology Bulletin, 23, 945-954. doi: $10.1177 / 0146167297239004$

Orbell, S., \& Sheeran, P. (1998). 'Inclined abstainers': A problem for predicting health related behaviour. British Journal of Social Psychology, 37, 151-165.

Orbell, S., \& Verplanken, B. (2010). The automatic component of habit in health behavior: Habit as cue-contingent automaticity. Health Psychology, 29, 374-383. doi: 10.1037/a0019596

Pashler, H., \& Harris, C. R. (2012). Is the replicability crisis overblown? Three arguments examined. Perspectives on Psychological Science, 7, 531-536. doi: 10.1177/1745691612463401

Payaprom, Y., Bennett, P., Alabaster, E., \& Tantipong, H. (2011). Using the Health Action Process Approach and implementation intentions to increase flu vaccine uptake in high risk Thai individuals: A controlled before-after trial. Health Psychology, 30, 492-500. doi: $10.1037 / \mathrm{a} 0023580$

Prestwich, A., Ayres, K., \& Lawton, R. (2008). Crossing two types of implementation intentions with a protection motivation intervention for the reduction of saturated fat intake: A randomized trial. Social Science \& Medicine, 67, 1550-1558. doi: 10.1016/j.socscimed.2008.07.019

Prestwich, A., Conner, M., Lawton, R., Bailey, W., Litman, J., \& Molyneaux, V. (2005). Individual and collaborative implementation intentions and the promotion of breast self-examination. Psychology \& Health, 20, 743-760. doi: 10.1080/14768320500183335

Prestwich, A., Conner, M. T., Lawton, R. J., Ward, J. K., Ayres, K., \& McEachan, R. R. C. (2012). Randomized controlled trial of collaborative implementation intentions targeting working adults' physical activity. Health Psychology, 31, 486-495. doi: 10.1037/a0027672

Prestwich, A., Lawton, R., \& Conner, M. (2003). The use of implementation intentions and the decision balance sheet in promoting exercise behaviour. Psychology and Health, 18, 707-721. doi: 10.1080/08870440310001594493

Prestwich, A., Perugini, M., \& Hurling, R. (2009). Can the effects of implementation intentions on exercise be enhanced using text messages? Psychology \& Health, 24, 677-687. doi: $10.1080 / 08870440802040715$ 
Quinn, J. M., Pascoe, A., Wood, W., \& Neal, D. T. (2010). Can't control yourself? Monitor those bad habits. Personality and Social Psychology Bulletin, 36, 499-511. doi: $10.1177 / 0146167209360665$

Rhodes, R. E., \& Dickau, L. (2012). Experimental evidence for the intention-behavior relationship in the physical activity domain: A meta-analysis. Health Psychology, 31, 724-727.

Ritchie, S. J., Wiseman, R., \& French, C. C. (2012). Replication, replication, replication. Psychologist, 25, 346-348.

Rogers, R. W. (1975). A protection motivation theory of fear appeals and attitude change. Journal of Psychology, 91, 93-114.

Rosenstock, I. M. (1974). Historical origins of the health belief model. Health Education Monographs, 2, 328-335.

Rutter, D. R., Steadman, L., \& Quine, L. (2006). An implementation intentions intervention to increase uptake of mammography. Annals of Behavioral Medicine, 32, 127-134. doi: $10.1207 / \mathrm{s} 15324796 \mathrm{abm} 3202 \_10$

Sandberg, T., \& Conner, M. (2009). A mere measurement effect for anticipated regret: Impacts on cervical screening attendance. British Journal of Social Psychology, 48, 221-236.

Scholz, U., Ochsner, S., \& Luszczynska, A. (in press). Comparing different boosters of planning interventions on changes in fat consumption in overweight and obese individuals: A randomized controlled trial. International Journal of Psychology. doi: 10.1080/00207594.2012.661061

Scholz, U., Schuz, B., Ziegelmann, J. R., Lippke, S., \& Schwarzer, R. (2008). Beyond behavioural intentions: Planning mediates between intentions and physical activity. British Journal of Health Psychology, 13, 479-494. doi: 10.1348/135910707x216062

Scholz, U., Sniehotta, F. F., Schuz, B., \& Oeberst, A. (2007). Dynamics in self-regulation: Plan execution self-efficacy and mastery of action plans. Journal of Applied Social Psychology, 37, 2706-2725.

Schuz, B., Wiedemann, A. U., Mallach, N., \& Scholz, U. (2009). Effects of a short behavioural intervention for dental flossing: Randomized-controlled trial on planning when, where and how. Journal of Clinical Periodontology, 36, 498-505. doi: 10.1111/j.1600051X.2009.01406.X

Schwarzer, R. (2001). Social-cognitive factors in changing health-related behavior. Current Directions in Psychological Science, 10, 47-51.

Schwarzer, R. (2008). Modeling health behaviour change: How to predict and modify the adoption and maintenance of health behaviors. Applied Psychology: An International Review, 57, 1-29.

Schwarzer, R., \& Luszczynska, A. (2008). How to overcome health-compromising behaviors The health action process approach. European Psychologist, 13, 141-151. doi: 10.1027/1016-9040.13.2.141

Schwerdtfeger, A. R., Schmitz, C., \& Warken, M. (2012). Using text messages to bridge the intention-behavior gap? A pilot study on the use of text message reminders to increase objectively assessed physical activity in daily life. Frontiers in Psychology, 3, 270. doi: 10.3389/fpsyg.2012.00270

Sheeran, P. (2002). Intention-behavior relations: A conceptual and empirical review. In W. Stroebe \& M. Hewstone (Eds.), European Review of Social Psychology (pp. 1-36). London: Wiley.

Sheeran, P., Milne, S., Webb, T. L., \& Gollwitzer, P. M. (2005). Implementation intentions and health behaviours. In M. Conner \& P. Norman (Eds.), Predicting health behaviour: Research and practice with social cognition models (2nd ed., pp. 276-323). Buckingham: Open University Press. 
Sheeran, P., \& Orbell, S. (1999a). Augmenting the Theory of Planned Behavior: Roles for anticipated regret and descriptive norms. Journal of Applied Social Psychology, 29, 2107-2142. doi: 10.1111/j.1559-1816.1999.tb02298.x

Sheeran, P., \& Orbell, S. (1999b). Implementation intentions and repeated behaviour: Augmenting the predictive validity of the theory of planned behaviour. European Journal of Social Psychology, 29, 349-369. doi: 10.1002/(SICI)10990992(199903/05)29:2/3<349::AID-EJSP931>3.0.CO;2-Y

Sheeran, P., \& Orbell, S. (2000). Using implementation intentions to increase attendance for cervical cancer screening. Health Psychology, 19, 283-289.

Sheeran, P., \& Silverman, M. (2003). Evaluation of three interventions to promote workplace health and safety: Evidence for the utility of implementation intentions. Social Science and Medicine, 56, 2153-2163.

Sheeran, P., Webb, T. L., \& Gollwitzer, P. M. (2005). The interplay between goal intentions and implementation intentions. Personality and Social Psychology Bulletin, 31, 87-98. doi: $10.1177 / 0146167204271308$

Sniehotta, F. F. (2009). Towards a theory of intentional behaviour change: Plans, planning and self-regulation. British Journal of Health Psychology, 14, 261-273. doi: 10.1348/135910708X389042

Sniehotta, F. F., Scholz, U., \& Schwarzer, R. (2005). Bridging the intention-behaviour gap: Planning, self-efficacy, and action control in the adoption and maintenance of physical exercise. Psychology and Health, 20, 143-160.

Sniehotta, F. F., Scholz, U., \& Schwarzer, R. (2006). Action plans and coping plans for physical exercise: A longitudinal intervention study in cardiac-rehabilitation. British Journal of Health Psychology, 11, 23-37.

Sniehotta, F. F., Scholz, U., Schwarzer, R., Fuhrmann, B., Kiwus, U., \& Voller, H. (2005). Long-term effects of two psychological interventions on physical exercise and selfregulation following coronary rehabilitation. International Journal of Behavioral Medicine, 12, 244-255. doi: 10.1207/s15327558ijbm1204_5

Sniehotta, F. F., Schwarzer, R., Scholz, U., \& Schuz, B. (2005). Action planning and coping planning for long-term lifestyle change: Theory and assessment. European Journal of Social Psychology, 35, 565-576. doi: 10.1002/ejsp.258

Solomon, R. L. (1949). An extension of control group design. Psychological Bulletin, 46, 137150. doi: $10.1037 / \mathrm{h} 0062958$

Strack, F., \& Deutsch, R. (2004). Reflective and impulsive determinants of social behavior. Personality and Social Psychology Review, 8, 220-247. doi: 10.1207/s15327957pspr0803_1

Sullivan, H. W., \& Rothman, A. J. (2008). When planning is needed: Implementation intentions and attainment of approach versus avoidance health goals. Health Psychology, 27, 438-444. doi: 10.1037/0278-6133.27.4.438

Tam, L., Bagozzi, R. P., \& Spanjol, J. (2010). When planning is not enough: The selfregulatory effect of implementation intentions on changing snacking habits. Health Psychology, 29, 284-292. doi: 10.1037/a0019071

Teng, Y., \& Mak, W. W. S. (2011). The role of planning and self-efficacy in condom use among men who have sex with men: An application of the Health Action Process Approach model. Health Psychology, 30, 119-128. doi: 10.1037/a0022023

Torregrossa, M. M., Quinn, J. J., \& Taylor, J. R. (2008). Impulsivity, compulsivity, and habit: The role of orbitofrontal cortex revisited. Biological Psychiatry, 63, 253-255. doi: 10.1016/j.biopsych.2007.11.014

Verplanken, B., Hofstee, G., \& Janssen, H. (1998). Accessibility of affective versus cognitive components of attitudes. European Journal of Social Psychology, 28, 23-35. 
von Suchodoletz, A., \& Achtziger, A. (2011). Intentions and their limits perspectives in psychological science. Social Psychology, 42, 85-92. doi: 10.1027/1864-9335/a000046

Webb, T. L., Christian, J., \& Armitage, C. J. (2007). Helping students turn up for class: Does personality moderate the effectiveness of an implementation intention intervention? Learning and Individual Differences, 17, 316-327. doi: 10.1016/j.lindif.2007.03.001

Webb, T. L., \& Sheeran, P. (2004). Identifying good opportunities to act: Implementation intentions and cue discrimination. European Journal of Social Psychology, 34, 407419.

Webb, T. L., \& Sheeran, P. (2006). Does changing behavioral intentions engender behavior change? A meta-analysis of the experimental evidence. Psychological Bulletin, 132, 249-268. doi: 10.1037/0033-2909.132.2.249

Webb, T. L., \& Sheeran, P. (2008). Mechanisms of implementation intention effects: The role of goal intentions, self-efficacy, and accessibility of plan components. British Journal of Social Psychology, 47, 373-395. doi: 10.1348/014466607X267010

Webb, T. L., Sheeran, P., \& Luszczynska, A. (2009). Planning to break unwanted habits: Habit strength moderates implementation intention effects on behaviour change. British Journal of Social Psychology, 48, 507-523. doi: 10.1348/014466608x370591

Webb, T. L., Sniehotta, F. F., \& Michie, S. (2010). Using theories of behaviour change to inform interventions for addictive behaviours. Addiction, 105, 1879-1892. doi: 10.1111/j.1360-0443.2010.03028.x

Wegner, D. M., Schneider, D. J., Carter, S. R., \& White, T. L. (1987). Paradoxical effects of thought suppression. Journal of Personality and Social Psychology, 53, 5-13. doi: 10.1037/0022-3514.53.1.5

Wiedemann, A. U., Lippke, S., Reuter, T., Ziegelmann, J. P., \& Schuz, B. (2011). The more the better? The number of plans predicts health behaviour change. Applied PsychologyHealth and Well Being, 3, 87-106. doi: 10.1111/j.1758-0854.2010.01042.x

Wiedemann, A. U., Lippke, S., \& Schwarzer, R. (2012). Multiple plans and memory performance: Results of a randomized controlled trial targeting fruit and vegetable intake. Journal of Behavioral Medicine, 35, 387-392. doi: 10.1007/s 10865-011-9364-2 
Table 1

Important Issues and Linked Research Priorities to Advance Knowledge on Planning

Interventions.

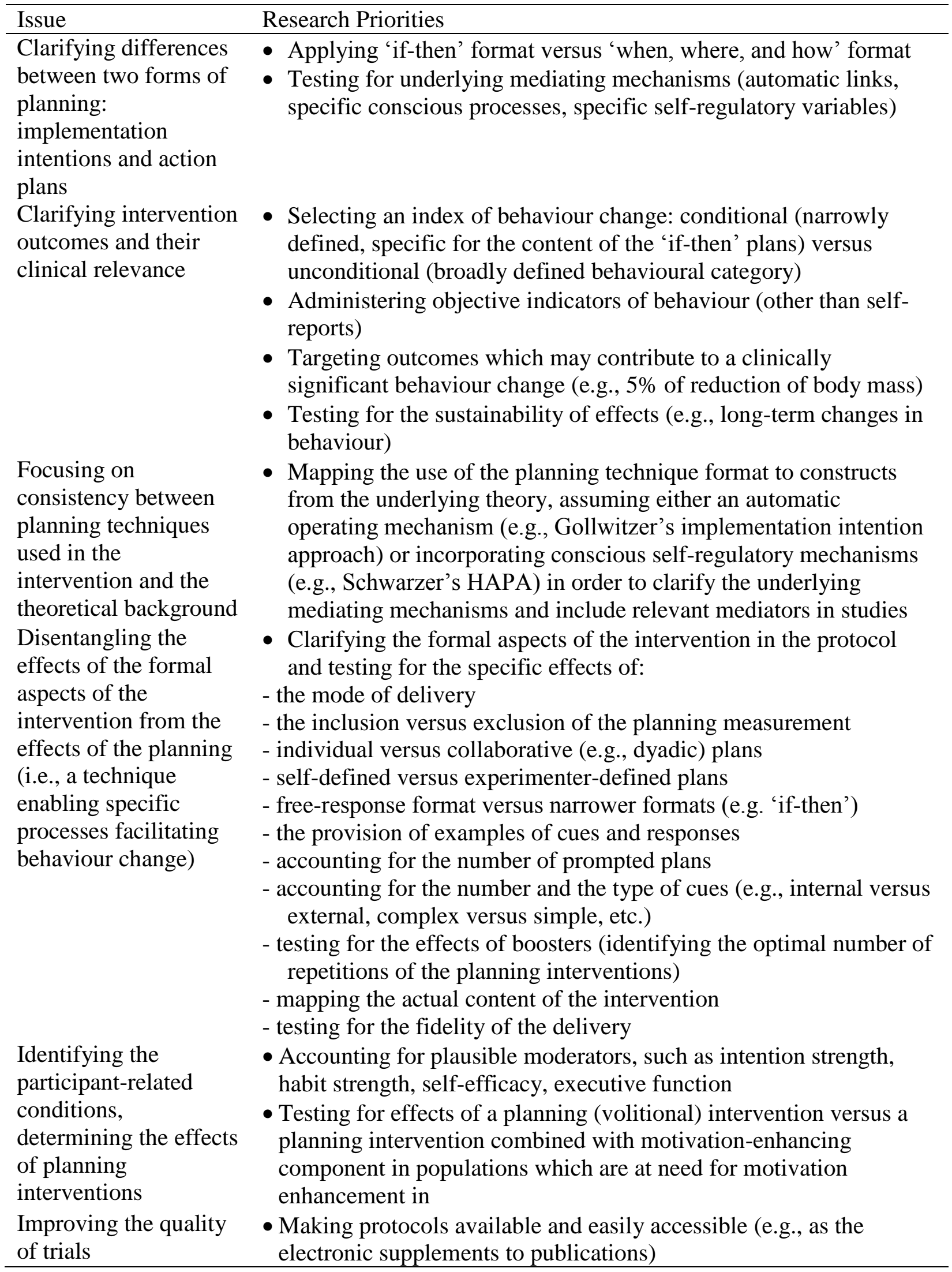


- Where applicable, applying Solomon design to account for 'mere measurement processes'

- Following Consort reporting guidelines and considering trial and protocol registration 